\title{
Stability of zero-pressure-gradient boundary layer distorted by unsteady Klebanoff streaks
}

\author{
NICHOLAS J. VAUGHAN AND TAMER A. ZAKI $\dagger$ \\ Department of Mechanical Engineering, Imperial College, London SW7 2AZ, UK \\ (Received 17 August 2010; revised 4 April 2011; accepted 11 April 2011; \\ first published online 31 May 2011)
}

The secondary instability of a zero-pressure-gradient boundary layer, distorted by unsteady Klebanoff streaks, is investigated. The base profiles for the analysis are computed using direct numerical simulation (DNS) of the boundary-layer response to forcing by individual free-stream modes, which are low frequency and dominated by streamwise vorticity. Therefore, the base profiles take into account the nonlinear development of the streaks and mean flow distortion, upstream of the location chosen for the stability analyses. The two most unstable modes were classified as an inner and an outer instability, with reference to the position of their respective critical layers inside the boundary layer. Their growth rates were reported for a range of frequencies and amplitudes of the base streaks. The inner mode has a connection to the TollmienSchlichting (T-S) wave in the limit of vanishing streak amplitude. It is stabilized by the mean flow distortion, but its growth rate is enhanced with increasing amplitude and frequency of the base streaks. The outer mode only exists in the presence of finite amplitude streaks. The analysis of the outer instability extends the results of Andersson et al. (J. Fluid Mech. vol. 428, 2001, p. 29) to unsteady base streaks. It is shown that base-flow unsteadiness promotes instability and, as a result, leads to a lower critical streak amplitude. The results of linear theory are complemented by DNS of the evolution of the inner and outer instabilities in a zero-pressure-gradient boundary layer. Both instabilities lead to breakdown to turbulence and, in the case of the inner mode, transition proceeds via the formation of wave packets with similar structure and wave speeds to those reported by Nagarajan, Lele \& Ferziger (J. Fluid Mech., vol. 572, 2007, p. 471).

Key words: boundary layer stability, transition to turbulence

\section{Introduction}

Transition to turbulence in boundary layers is often classified as orderly or bypass. The early stages of orderly transition are characterized by the amplification of twodimensional Tollmien-Schlichting ( $\mathrm{T}-\mathrm{S}$ ) instability waves. The primary $\mathrm{T}-\mathrm{S}$ waves develop a three-dimensional secondary instability, and subsequently break down to turbulence (Kleiser \& Zang 1991). When the proceedings of transition deviate from this description, the breakdown mechanism is termed bypass transition (Morkovin 1969). This terminology is general, and encompasses a variety of scenarios where $\mathrm{T}-\mathrm{S}$ waves are absent or play a less prominent role (Morkovin, Reshotko \& Herbert 1994), for example due to surface roughness or free-stream vortical or acoustic forcing 
(Reshotko 1976; Saric, Reed \& Kerschen 2002). The term bypass has, however, often been used in reference to transition in boundary layers exposed to free-stream vortical disturbances.

Bypass transition also involves the amplification of a primary disturbance, a secondary instability and nonlinear breakdown. In response to forcing by freestream turbulence, a laminar boundary layer develops high-amplitude, low-frequency perturbations which are known as streaks, or Klebanoff modes (Klebanoff 1971; Kendall 1991). Their spanwise size is of the order of the boundary-layer thickness, they are elongated in the flow direction and are dominated by their streamwise component, $u$. The streaky boundary layer subsequently undergoes a secondary instability that intensifies and leads to the formation of turbulent spots, which lead to turbulence.

The amplification of Klebanoff streaks is well understood, and has been the subject of a wealth of analytical, experimental and numerical studies (e.g. Hultgren \& Gustavsson 1981; Westin et al. 1994; Jacobs \& Durbin 2001). Their elongated appearance is explained by the rapid distortion theory (Phillips 1969), and their spanwise scale can be predicted using transient growth analysis (Butler \& Farrell 1992). The physical mechanism for their amplification is displacement of mean momentum, or lift-up (Landahl 1980).

On the other hand, the influence of Klebanoff streaks on boundary-layer stability remains the subject of active research. Two main effects have been identified. (a) In bypass transition, streaks above a critical amplitude develop a secondary instability (e.g. Andersson et al. 2001). (b) Klebanoff streaks can also alter the growth rate of instabilities of the Tollmien-Schlichting type. Both a stabilizing and a destabilizing influence have been suggested in the literature (e.g. Cossu \& Brandt 2002; Liu, Zaki $\&$ Durbin 2008b). These two issues are addressed herein using secondary instability analysis.

\subsection{The instability due to Klebanoff streaks in bypass transition}

The notion that a secondary instability due to Klebanoff streaks plays a role in the inception of spots in bypass transition can be traced back to work on Görtler vortices. Swearingen \& Blackwelder (1987) identified two types of secondary instability of Görtler flow, which were distinguished by their symmetry with respect to the underlying streak: The varicose instability has a symmetric wall-normal and streamwise velocity pattern with respect to the streak. The sinuous mode is antisymmetric with respect to the base flow, with transversely oscillating perturbation streamlines. The sinuous mode was more rapidly growing, and was related to spanwise shear, whereas the varicose mode was associated with wall-normal shear (Saric 1994; Asai, Minagawa \& Nishioka 2002).

In flat-plate boundary layers, streaks can also cause the flow to become prone to secondary instabilities. Matsubara \& Alfredsson (2001) experimentally observed that streaks undergo a high-frequency oscillation prior to breakdown into turbulent spots. In direct numerical simulation (DNS), Jacobs \& Durbin (2001) showed that turbulent spots are preceded by an interaction of the lifted low-speed streaks with free-stream eddies. They did not describe the interaction as a streak instability, but the results presented herein support such a view. The same mechanism, originating near the edge of the boundary layer, was documented by Hernon, Walsh \& McEligot (2007b) in their experimental investigation of bypass transition.

Linear theory has been applied to the study of secondary instability of boundary layers distorted by Klebanoff streaks. Andersson et al. (2001) carried out the first inviscid, secondary instability analysis of the so-called 'optimal' boundary-layer 
streaks. They reported critical streak amplitudes of $26 \%$ and $37 \%$ for the sinuous and varicose instability modes, respectively. These modes were subsequently prescribed as inlet perturbations in the DNS of Brandt \& Henningson (2002). The simulations affirmed the amplification of the secondary instability modes, which resulted in breakdown to turbulence.

The sinuous and varicose instabilities were also identified, albeit empirically, in experiments (Mans et al. 2005; Mans, de Lange \& van Steenhoven 2007) and simulations (Brandt, Sclatter \& Henningson 2004) of bypass transition. However, a discrepancy remains between the critical streak amplitude based on stability theory and experimental observations. While the former predicts a threshold amplitude of $26 \%$, Arnal \& Juillen (1978) showed that modest streak intensities with $u_{r m s} \sim 5-7 \%$ were sufficient for transition; Mandal, Venkatakrishnan \& DEY (2010) report $u_{r m s}=9 \%$ before transition.

Two explanations address the disagreement between the theoretical and measured thresholds for instability, and are both important in mitigating this discord. (i) The first points to the deviation of instantaneous streak amplitudes from the root-mean-square values often reported upstream of transition (Hernon et al. 2007b; Nolan, Walsh $\&$ McEligot 2010). (ii) The second explanation, which is the focus of the current work, is motivated by empirical evidence of the importance of streak unsteadiness. For example, based on their simulations, Brandt et al. (2004) asserted that varicose breakdown is initiated where the streamwise velocity perturbation changes direction between passing streaks. Also, Mans et al. (2007) studied the sinuous instability mechanism experimentally, and identified its origin near the upstream terminus of the low-speed streak. This location corresponds to the maximum rate of change of the streaky velocity perturbation.

The current work applies Floquet analysis in order to examine the stability of boundary-layer streaks. Unlike the analysis of Andersson et al. (2001), which considered the linearly optimal, steady streaks, our base flow is obtained by simulating the boundary-layer response to free-stream vortical disturbances. Therefore, our streak ansatz represents the flow in the simulations of Jacobs \& Durbin (2001) and Zaki \& Durbin (2005) more faithfully. In addition, our free-stream disturbance is unsteady in order to assess the role of streak frequency on boundary-layer stability. It is well known that free-stream turbulence forces a spectrum of streak frequencies inside the boundary layer. The lowest frequency streaks amplify most, and dominate the disturbance field inside the shear. Whether these streaks are most unstable is not known, and will be addressed herein.

\subsection{The influence of streaks on Tollmien-Schlichting-type instabilities}

Previous studies have provided evidence that streaks can modify the growth rate of instability modes that belong to the classical boundary-layer eigenspectrum, for example $\mathrm{T}-\mathrm{S}$ waves. However, experimental, numerical and theoretical efforts have not yielded a unified view as to whether this modification is stabilizing or destabilizing.

Boiko et al. (1994) experimentally observed that boundary layers subject to freestream turbulence (FST) levels of $1.5 \%$ can support growing T-S waves. Their growth rate, however, was lower than in an undisturbed Blasius flow. Further evidence is provided from numerical simulations (Fasel 2002; Cossu \& Brandt 2002; Liu, Zaki \& Durbin 2008a). In these studies, moderate streak amplitudes were reported to reduce the growth rate of primary T-S waves - although a secondary instability can be promoted. The stabilization effect was attributed to nonlinear modification of the mean velocity profile, which became fuller than the Blasius solution and resembled 
a favourable pressure gradient boundary layer. In addition, the spanwise Reynolds stress associated with the streak profile made a stabilizing contribution to the energy balance of Tollmien-Schlichting-type waves (Cossu \& Brandt 2004).

Contrary to the above literature, some studies have reported that $\mathrm{T}-\mathrm{S}$ waves become more unstable in the presence of streaks. For instance, Kendall (1990) showed that the growth of T-S waves depends on the level of FST and, in turn, on the amplitude of the Klebanoff streaks inside the boundary layer. Noise levels around $0.2 \%$ brought about instabilities which resembled $\mathrm{T}-\mathrm{S}$ waves, but grew more rapidly than in the absence of free-stream turbulence.

In addition to the influence on viscous $\mathrm{T}-\mathrm{S}$ waves, theoretical analyses have predicted that streaks can destabilize oblique Rayleigh waves. For example, Goldstein \& Wundrow (1995) studied boundary-layer stability in the presence of weak streamwise vortices, which generate a streamwise velocity distortion. They showed that this flow supports three-dimensional, Rayleigh-type instabilities which can far exceed the growth rates of classical Tollmien-Schlichting waves. Their analysis assumed that the streaks were much wider in the span than the boundary-layer thickness. More recently, Wu \& Choudhari (2003) proposed that streak unsteadiness can play an important destabilizing role.

An interesting case of transition in the presence of streaks and instability wave packets was reported by Nagarajan et al. (2007). They simulated transition due to FST in boundary layers over flat plates with slender and blunt leading edges. At moderate free-stream turbulence intensity, the slender leading edge did not play a significant role: Breakdown of the low-speed streaks was consistent with the mechanism illustrated by Jacobs \& Durbin (2001). However, downstream of their blunt leading edge, Nagarajan et al. (2007) observed the amplification of wave packets near the wall, which caused breakdown to turbulence. The appearance of these wave packets followed the interaction of strong free-stream vortices with the blunt leading edge. This mechanism was therefore absent from the simulations by Jacobs \& Durbin (2001), which excluded the leading-edge region. The origin of the wave packet was investigated by Goldstein \& Sescu (2008), and was attributed to an inviscid instability of the velocity profile. The near-wall inflectional profile was caused by low-frequency unsteadiness introduced near the leading edge. Their findings therefore affirm that streak unsteadiness can have a significant effect. However, they adopted a highReynolds-number asymptotic approach and, as indicated by Goldstein \& Sescu (2008), the asymptotic approach 'may not apply at the more moderate Reynolds numbers'.

Near-wall boundary-layer instability will be examined herein, when the streaks are caused by free-stream vortical disturbances, at moderate Reynolds number. The viscous analysis will address the influence of the base streak unsteadiness and amplitude, and the connection to classical Tollmien-Schlichting waves in the limit of vanishing streak amplitude.

The preceding discussion summarizes two potential instabilities of streaky boundary layers: (i) an outer mechanism which acts on the base streaks and (ii) an inner mechanism which was manifested as near-wall instability wave packets in the simulations by Nagarajan et al. (2007). In this paper, we apply viscous stability theory to zero-pressure-gradient boundary layers distorted by Klebanoff streaks. Each streak distortion is induced by a free-stream vortical mode with prescribed frequency and amplitude, in order to emulate the influence of free-stream turbulence. The base flow is computed using DNS, and therefore non-parallelism and nonlinear effects are taken into account. The velocity profile is extracted at the location where the streak 
amplitude is maximum, and the analysis is repeated for a range of streak amplitudes and frequencies. A description of the base flow and the stability analysis is provided in $\S 2$. Two types of instability modes are identified, and their dependence on the amplitude and frequency of Klebanoff streaks is discussed in $\S 3$. Direct numerical simulations of the boundary-layer response to these instabilities are reported in $\S 4$, and concluding remarks are presented in $\S 5$.

\section{Theoretical formulation}

\subsection{The base flow}

The influence of streaks on the stability of the boundary layer can be formulated as a secondary instability problem. In such an approach, the base flow at the target location for the analysis is a superposition of a boundary-layer profile, $U_{0}(y)$, and a saturated streak $u_{1}(y, z, t)$,

$$
u_{2}(y, z, t)=U_{0}(y)+u_{1}(y, z, t) .
$$

Various base flow ansätze are possible, and the appropriate choice can be motivated by a discussion of previous work. Liu et al. $(2008 a, b)$ used a Blasius profile and a linear streak obtained from the solution to the Squire equation. We have carried out our initial analyses using a similar ansatz. However, we subsequently adopted a more 'realistic' profile due to the reasons discussed below.

Boundary-layer streaks are elongated in the streamwise direction and therefore non-parallel effects become significant in determining their amplitude. The spanwise size of the streaks is of the order of the boundary-layer thickness and, as a result, both wall-normal and cross-flow ellipticity must be taken into account in predicting their evolution. In order to account for these effects, the boundary region equations can be used (Goldstein \& Leib 1993; Leib, Wundrow \& Goldstein 1999). However, the linear formulation is not sufficient because the streaks reach a high amplitude. Nonlinear effects become important and can, for example, cause a deviation of the mean flow profile from the Blasius solution. This zero-frequency distortion of the base flow can, in turn, alter the growth rates of Tollmien-Schlichting waves (see e.g. Cossu $\&$ Brandt 2002). In addition, Andersson et al. (2001) demonstrated that it is not sufficient to describe the streaks by their fundamental spanwise components alone. Instead, higher spanwise harmonics, i.e. the full nonlinear streaks, are required in the representation of the base state in order to correctly predict the dominant instability mode.

The base flow for the current analysis is obtained using direct numerical simulations of the Navier-Stokes equations for a zero-pressure-gradient boundary layer. A schematic of the computational domain is shown in figure 1, and the details of the computational algorithm are described in $\S 4$. The inflow to the DNS domain, at $x=x_{0}$, is a superposition of the Blasius profile $\boldsymbol{v}_{B}$, and an unsteady, free-stream vortical disturbance with amplitude $A_{v}$,

$$
\begin{aligned}
\boldsymbol{v}_{0}\left(x=x_{0}, y, z, t\right)=\boldsymbol{v}_{B}\left(x_{0}, y\right) & +A_{v} \operatorname{Re}\left\{\hat{u}_{1}\left(x_{0}, y\right) \cos \left(k_{z} z\right) \mathrm{e}^{\mathrm{i}(-\omega t)}\right\} \boldsymbol{e}_{x} \\
& +A_{v} \operatorname{Re}\left\{\hat{v}_{1}\left(x_{0}, y\right) \cos \left(k_{z} z\right) \mathrm{e}^{\mathrm{i}(-\omega t)}\right\} \boldsymbol{e}_{y} \\
& -A_{v} \operatorname{Im}\left\{\hat{w}_{1}\left(x_{0}, y\right) \sin \left(k_{z} z\right) \mathrm{e}^{\mathrm{i}(-\omega t)}\right\} \boldsymbol{e}_{z} .
\end{aligned}
$$

An example of the inflow mode and the boundary-layer response is shown in figure 2. The inlet mode belongs to the continuous branch of the boundary-layer eigenspectrum, and is monochromatic with frequency $\omega$ and spanwise wavenumber, $k_{z}$. The inflow plane is upstream of the zone of the boundary region equations denoted 


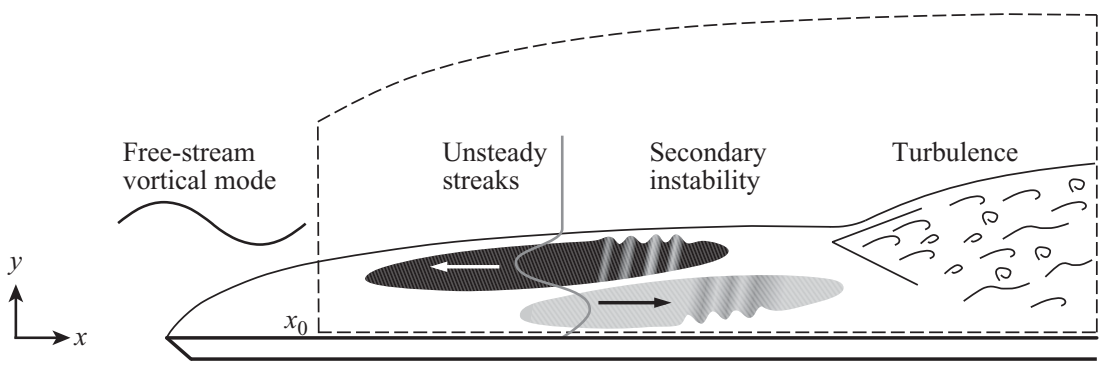

FiguRE 1. Schematic of the generation of streaks due to free-stream vortical forcing, their secondary instability, and breakdown to turbulence. The domain used in the DNS is marked by the dashed line.

(a)

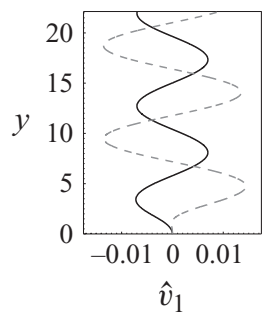

(b)

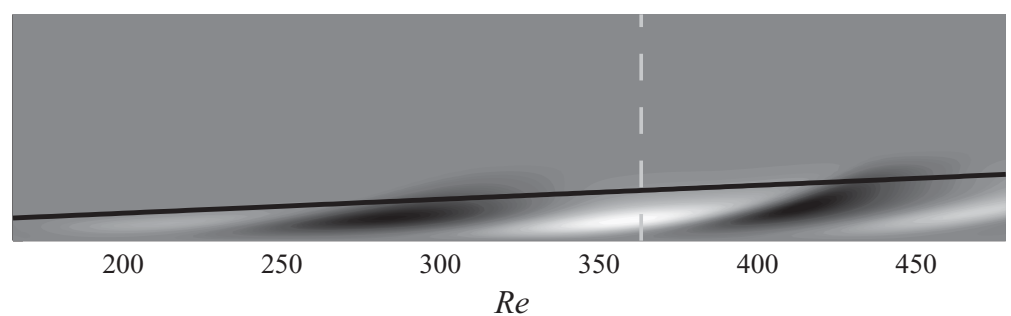

FiguRE 2. The boundary-layer response to forcing by a single, unsteady free-stream vortical mode, $F=60$. (a) $v$ component of the free-stream forcing mode; $(b)$ side view of the $u$-perturbation field, $-0.20<u_{1}<0.20$. Light and dark contours mark positive and negative velocity perturbations, respectively. The $y$-coordinate is normalized by the Blasius length scale at the target Reynolds number, $R e=360$, where the stability analyses are carried out. This location is marked by the dashed line.

III in Leib et al. (1999). Since our flow is computed using DNS, both non-parallel and nonlinear effects are fully captured, and the streak profiles downstream include higher harmonics of the inlet $\omega$ and $k_{z}$. In this and the following section, all variables are made non-dimensional using the free-stream speed $U_{\infty}$ and the Blasius length scale. The Reynolds number is therefore $R e \equiv \sqrt{U_{\infty} x / v}$.

The parameters for our stability analyses are motivated by the DNS of bypass transition at $T_{u}=3 \%$ by Jacobs \& Durbin (2001). The Reynolds number at the inlet to our computational domain is the same as in their simulation, $R e=163$. Every unsteady, free-stream vortical mode of interest was simulated independently. Their frequencies, $F \equiv 10^{6} \omega / R e<60$, and spanwise wavenumber, $k_{z}=0.71$, are representative of the streaks with the highest energy content in the DNS of Jacobs \& Durbin (2001). Each inlet mode caused the downstream amplification of streaks, followed by viscous decay. Figure 3 shows an example of streak amplitude, $A_{u} \equiv \max \left|u_{1}\right|$, versus Reynolds number. The peak streak amplitude is reached near $R e=360$, and this location is selected for our stability analyses (see marked location in figures 2 and 3). This target Reynolds number also lies within the range $350<R e<400$, where Jacobs \& Durbin (2001) observed the initial stages of streak interactions that lead to breakdown to turbulence.

Since the streaks reach high amplitude, nonlinear terms in the DNS are appreciable. As a result, a simple linear relationship between the inlet condition and the downstream streak amplitude is not possible. Instead, the amplitude of the inlet 


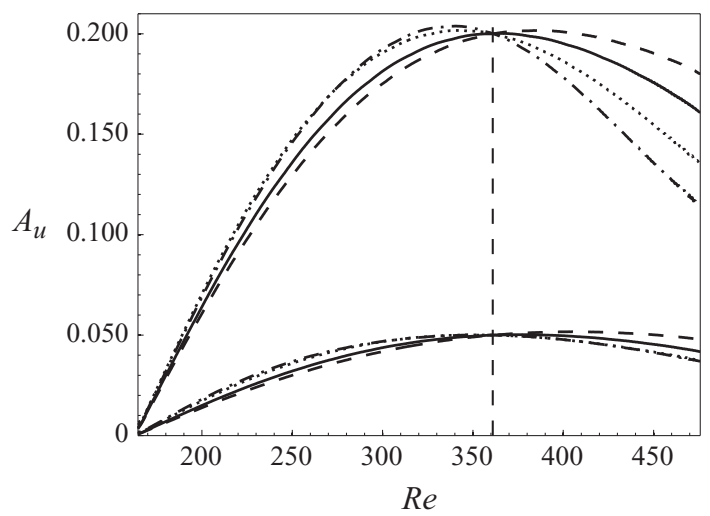

FIGURE 3. The evolution of the streak amplitude $A_{u}$ is plotted versus the Reynolds number, for streak frequencies,$-- F=0 ;-, F=20 ; \cdots, F=40 ;-\cdot-, F=60$. Two maximum streak amplitudes, $A_{u}=\{5,20\} \%$, at the target location $R e=360$ are shown.

free-stream disturbance was iteratively varied, and the computations were repeated in order to achieve the desired streak amplitudes, $A_{u}=\{5,10,15,20\} \%$, at the target Reynolds number. These values of $A_{u}$ span the range of interest, based on both computer and physical experiments (Westin et al. 1994; Matsubara \& Alfredsson 2001; Jacobs \& Durbin 2001).

The boundary-layer profile is computed at the target Reynolds number using spanwise- and time-averaging, $U_{0}=\left\langle\overline{u_{2}}\right\rangle$. The streak profile is also evaluated at the target location, and is decomposed into its Fourier modes which are harmonics of the inlet forcing $\omega$ and $k_{z}$,

$$
u_{1}(y, z, t)=\sum_{m=0}^{M} \sum_{n=0}^{N} \operatorname{Re}\left\{\hat{u}_{1}^{m, n}(y) \mathrm{e}^{\mathrm{i}\left(m \omega t+n k_{z} z\right)}\right\} .
$$

Note that the $(0,0)$ component is already included in the mean flow $U_{0}$ and, as a result, $u_{1}^{0,0}$ is zero. In the stability analysis, the representation of the streaks includes sufficient Fourier components in order to ensure that at least $98 \%$ of its kinetic energy is captured. Most of the energy is in the fundamental mode, and therefore it is sufficient to truncate the Fourier series at $M=2, N=2$; this is verified in Appendix A.

An example of the boundary-layer profile and the Fourier components of the streak is shown in figure 4 . These profiles are extracted at the marked location in figures 2 and 3. The mean velocity exceeds the Blasius profile near the wall, and shows a deficit towards the edge of the boundary layer. The same trend was observed in experiments of bypass transition (e.g. Mandal et al. 2010). The deviation of $U_{0}$ from Blasius increases with the amplitude of the base streaks. Here, the maximum base-flow modification is less than $4.35 \%$ when the base streaks are unsteady, and less than $5.24 \%$ in case of steady base streaks. This level of distortion can cause significant changes to the near-wall curvature of the velocity profile, which, in turn, can affect the boundary-layer stability (Wu \& Choudhari 2003). Results from our stability analyses will compare the effects of using the mean flow from the DNS and the Blasius profile, in order to quantify the influence of the base-flow modification. The fundamental mode in the Fourier expansion of the base streak, $\hat{u}_{1}^{1,1}$, is shown in figure $4(b)$, and resembles a Klebanoff mode. The first harmonic in the spanwise direction, $\hat{u}_{1}^{1,2}$, is plotted in figure $4(c)$, and is significantly less energetic. 

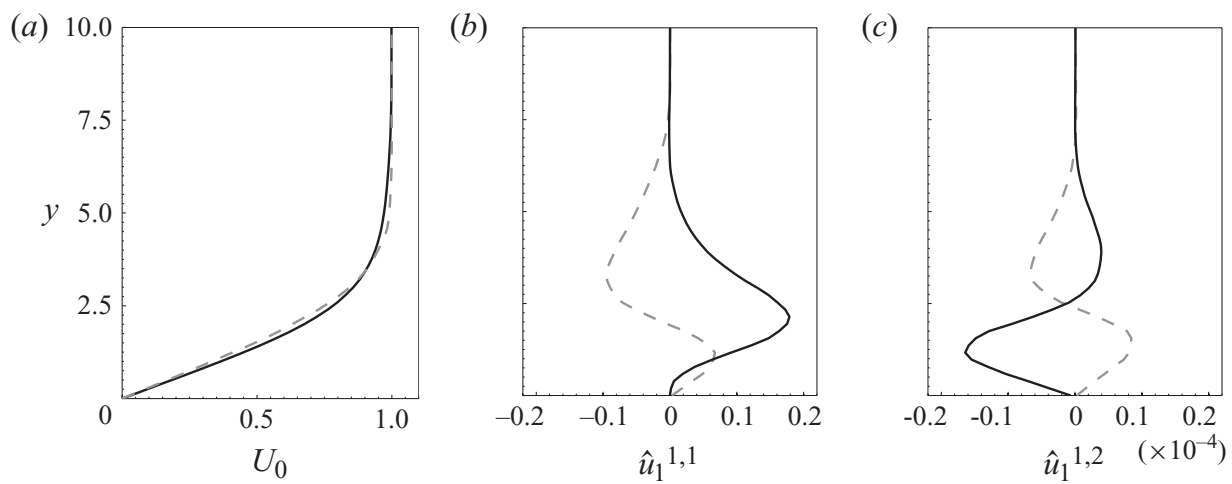

FIGURE 4. The wall-normal profile of the streamwise velocity at the target Reynolds number, $R e=360$. The streak amplitude is $A_{u}=20 \%$ and the frequency is $F=60$. (a) The mean flow computed using time-averaging of the DNS (-) is plotted along with the Blasius solution (---). (b) The fundamental and $(c)$ the spanwise harmonic components in the Fourier representation of the base streak (2.3). The solid and dashed lines correspond to the real and imaginary parts of $\hat{u}_{1}^{m, n}$.

\subsection{Secondary instability}

The secondary stability equations are derived by considering linear perturbations to the base state, described in $\S 2.1$. Evidence from previous DNS and experiments indicate that the secondary instability has a short streamwise wavelength and, therefore, non-parallel effects can be ignored. A local velocity profile is extracted at the target Reynolds number, and the perturbed state is expressed as a summation of the streamwise base flow and a three-dimensional disturbance

$$
\boldsymbol{v}(x, y, z, t)=\left\{U_{0}(y)+u_{1}(y, z, t)\right\} \boldsymbol{e}_{x}+B \boldsymbol{v}_{3}(x, y, z, t),
$$

where $B$ is sufficiently small for linearization. The secondary perturbation problem is derived by substituting (2.4) in the Navier-Stokes equations, subtracting the equations for the base state $u_{2}$ and neglecting terms of order $B^{2}$. By taking the curl of the velocity perturbation equations, the secondary instability problem is expressed in terms of the vorticity perturbation

$$
\left(\frac{1}{R e} \nabla^{2}-\frac{\partial}{\partial t}\right) \omega_{3}-\left(v_{2} \cdot \nabla\right) \omega_{3}-\left(v_{3} \cdot \nabla\right) \omega_{2}+\left(\omega_{2} \cdot \nabla\right) v_{3}+\left(\omega_{3} \cdot \nabla\right) v_{2}=0 .
$$

where $\omega \equiv(\xi, \eta, \zeta)^{T}$ is the vorticity vector.

In analogy to the Orr-Sommerfeld and Squire primary perturbation equations, a pair of equations is derived for the secondary disturbance in terms of $\nabla^{2} v_{3}$ and $\eta_{3}$. Since $w_{3}$ cannot be eliminated from the secondary instability problem, the continuity equation is required in order to complete the system

$$
\begin{aligned}
& \frac{\partial^{2} U_{0}}{\partial y^{2}} \frac{\partial v_{3}}{\partial x}+\left(\frac{1}{R e} \nabla^{2}-\frac{\partial}{\partial t}-U_{0} \frac{\partial}{\partial x}-u_{1} \frac{\partial}{\partial x}\right) \nabla^{2} v_{3}+\left(-\left[\frac{\partial^{2} u_{1}}{\partial y \partial z}+\frac{\partial u_{1}}{\partial z} \frac{\partial}{\partial y}\right] \eta_{3}\right. \\
& \left.+\left[\frac{\partial \xi_{3}}{\partial x}-\frac{\partial \zeta_{3}}{\partial z}\right] \frac{\partial u_{1}}{\partial z}+\frac{\partial v_{3}}{\partial x}\left[\frac{\partial^{2} u_{1}}{\partial y^{2}}-\frac{\partial^{2} u_{1}}{\partial z^{2}}\right]+\left[\frac{\partial u_{3}}{\partial z}+\frac{\partial w_{3}}{\partial x}\right] \frac{\partial^{2} u_{1}}{\partial y \partial z}\right)=0,
\end{aligned}
$$




$$
\begin{gathered}
\left(\frac{1}{R e} \nabla^{2}-\frac{\partial}{\partial t}\right) \eta_{3}-\frac{\partial \eta_{3}}{\partial x} U_{0}-\frac{\partial v_{3}}{\partial z} \frac{\partial U_{0}}{\partial y} \\
-\left(\frac{\partial \eta_{3}}{\partial x} u_{1}+v_{3} \frac{\partial^{2} u_{1}}{\partial y \partial z}+w_{3} \frac{\partial^{2} u_{1}}{\partial z^{2}}-\frac{\partial v_{3}}{\partial y} \frac{\partial u_{1}}{\partial z}+\frac{\partial v_{3}}{\partial z} \frac{\partial u_{1}}{\partial y}\right)=0 \\
\frac{\partial u_{3}}{\partial x}+\frac{\partial v_{3}}{\partial y}+\frac{\partial w_{3}}{\partial z}=0 .
\end{gathered}
$$

Homogeneous boundary conditions are enforced on $u_{3}, v_{3}, w_{3}$, and on $\partial v_{3} / \partial y$ at the wall and in the free stream.

The coefficients of the secondary instability problem (2.6)-(2.8) are homogeneous in $x$. As a result, a normal mode assumption can be invoked in that dimension. Further simplifications are possible, and depend on the form of the base flow, $u_{2}(y, z, t)$. Since the streaks are periodic in the spanwise direction, the Floquet theory must be invoked for the $z$-dimension. As regards the time dependence of the base state, two classes of analyses will be considered. The first approach will invoke a quasi-steady assumption where the stability analysis is repeated for each phase of the unsteady streak, $u_{1}=u_{1}(y, z ; t)$. In this case, a normal mode assumption is also possible in time. In the second class, the temporal periodicity of $u_{1}(y, z, t)$ is retained in the definition of the base flow, and a Floquet expansion is therefore required in time as well.

The quasi-steady assumption has been invoked in previous stability analyses of streaky base flows. For example, Wu \& Choudhari (2003) adopted such an approach. However, in their discussion, they have also acknowledged that the intermittent instabilities' predicted by the quasi-steady assumption cannot be directly related to a global Floquet instability of the time-periodic base flow. The limitations of the quasi-steady assumption were discussed by Kerczek \& Davis (1974), who studied the linear stability of oscillatory Stokes layers. They reasoned that the quasi-steady approximation becomes inappropriate around the critical conditions for instability, where the time scale of amplification is very long and unstable base profiles might propagate away before instabilities could grow locally. Therefore, a Floquet expansion in time is required in order to accurately determine the stability of the time-periodic base flow. For example, Blennerhassett \& Bassom (2006) studied the instability of an oscillatory Stokes layer with a Floquet expansion in time, and Luo \& Wu (2010) compared the results to their direct numerical simulations of the same base flow at different Reynolds numbers. At subcritical Reynolds numbers, some phases of the base flow were instantaneously unstable, but others were stable. However, the neutral curve calculated from the Floquet analysis correctly predicted the critical conditions calculated by the DNS.

The Floquet analysis in time can be motivated further by comparing the frequency of the base streaks and that of the classical boundary-layer instability. It is known that the most energetic streaks lie in the frequency range $0<F<100$ (Jacobs \& Durbin 2001). Also, in the absence of streaks, the Blasius profile at the target Reynolds number can sustain viscous instability waves in the frequency range $137<F<240$. Although these modes have a weak growth rate, parametric excitation by the streaks is possible, and must be taken into account since it can lead to enhanced growth rates. Figure 5 shows this relationship between the neutral curve of the Blasius flow, and the energy spectrum inside the boundary layer from DNS of bypass transition at the same conditions as the work of Jacobs \& Durbin (2001). 


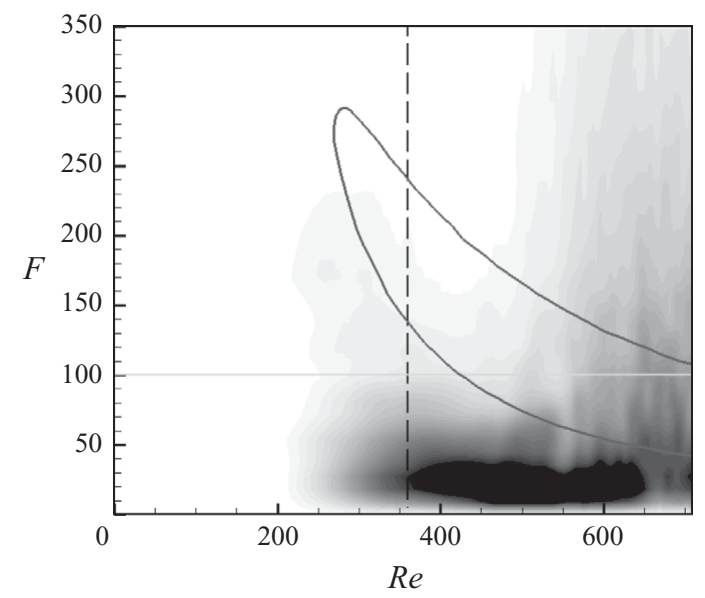

FigURE 5. The neutral curve for a Blasius boundary layer is overlaid on the energy spectrum inside the boundary layer from DNS of bypass transition at the same conditions as the work of Jacobs \& Durbin (2001). The target Reynolds number is marked by the dashed vertical line.

The secondary instability $\boldsymbol{v}_{3}$ can be expressed in the general form,

$$
\boldsymbol{v}_{3}(x, y, z, t)=\tilde{\boldsymbol{v}}_{3}(y, z, t) \mathrm{e}^{\mathrm{i}(\alpha x+\gamma z)+\sigma t} .
$$

In the temporal stability problem, both $\alpha$ and $\gamma$ are real parameters, and $\sigma$ is the complex eigenvalue. For the unsteady base flow, $\tilde{\boldsymbol{v}}_{3}(y, z, t)$ is periodic in both $z$ and $t$,

$$
\tilde{\boldsymbol{v}}_{3}(y, z, t)=\tilde{\boldsymbol{v}}_{3}\left(y, z+2 \pi / k_{z}, t\right)=\tilde{\boldsymbol{v}}_{3}(y, z, t+2 \pi / \omega) .
$$

The perturbation can therefore be replaced by its Fourier expansion in $t$ and $z$, and $\boldsymbol{v}_{3}$ has the form

$$
\boldsymbol{v}_{3}=\mathrm{e}^{\left(\mathrm{i} \alpha x+\sigma_{r} t\right)} \sum_{m=-\infty}^{\infty} \sum_{n=-\infty}^{\infty} \boldsymbol{v}_{m, n}(y) \mathrm{e}^{\mathrm{i}\left(\left[n k_{z}+\gamma\right] z+\left[m \omega+\sigma_{i}\right] t\right)},
$$

where $\gamma$ and $\sigma_{i}$ have been absorbed in the summation. The eigenvalue problem is formulated by substituting (2.10) into the governing equations (2.6)-(2.8). The growth rate of the instability is given by the real part of the eigenvalue $\sigma_{r}$, and the imaginary part $\sigma_{i}$ determines the frequency offset which is added to each Fourier component in the expansion. Note that when the base flow is assumed to be quasi-steady, $\omega=0$ and the frequency of the instability is determined by $\sigma_{i}$ solely.

In the spanwise direction, $\gamma$ is the detune factor which need only be considered over the range $|\gamma| \leqslant(1 / 2) k_{z}$. Larger values of $\gamma$ can be recast within the range, given a renumbering of the Fourier modes in the expansion. Fundamental and subharmonic resonance in the spanwise direction correspond to $\gamma=0$ and $\gamma=(1 / 2) k_{z}$, respectively. Other values of $\gamma$ correspond to detuned modes.

Due to the spanwise symmetry of the base flow, the eigenvalue problems associated with the sinuous and varicose components of the expansion (2.10) are decoupled, and each is solved independently in order to reduce the overall computational cost. The terminology sinuous and varicose refers to the appearance of the perturbation streamlines in the horizontal $(x-z)$ plane. Here, symmetry is with respect to the lowspeed streak, in keeping with the convention of Andersson et al. (2001). The sinuous 


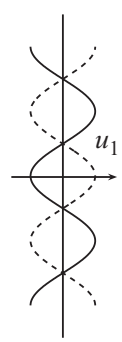

(a)
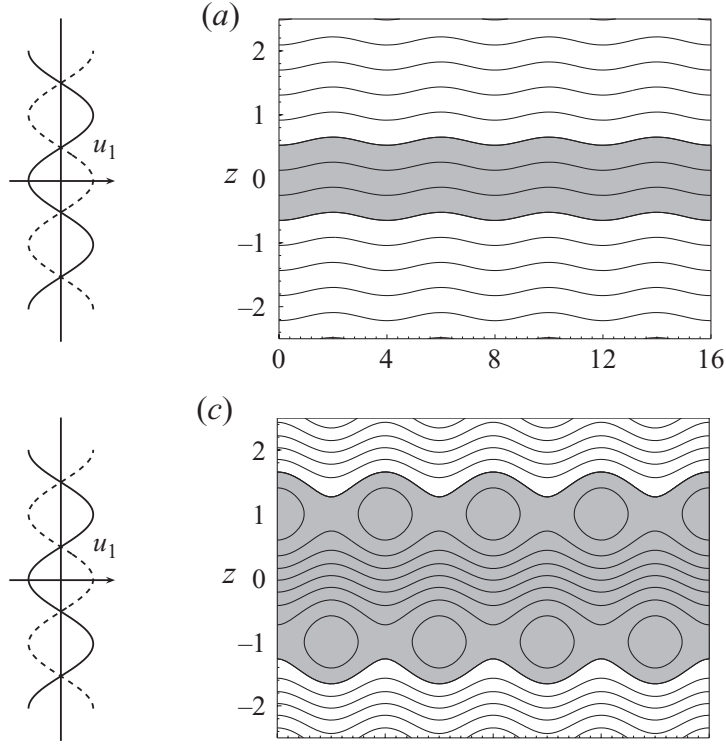

(c)

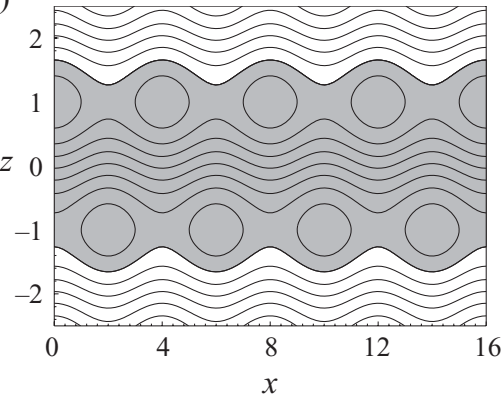

(b)

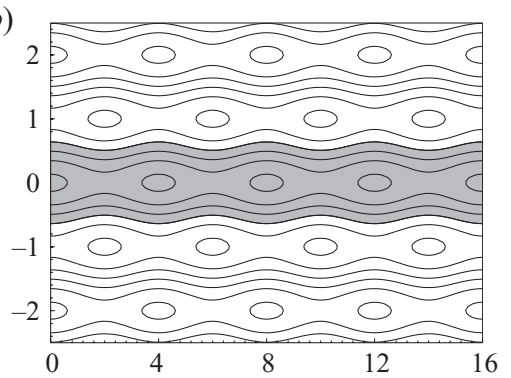

(d)

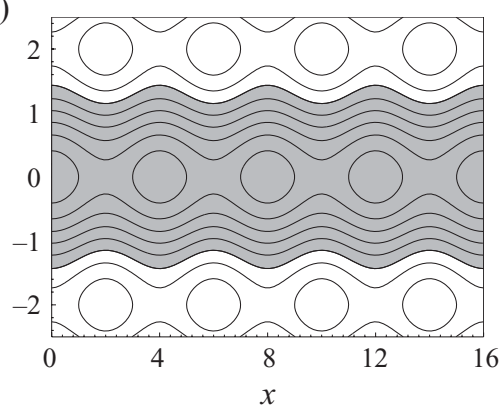

FIGURE 6. On the left, the spanwise variation of the streak amplitude is shown at two instances in time, which are exactly out of phase. The four sub-plots show streamlines of the perturbation field in the $(x-z)$ plane for $(a)$ the spanwise fundamental, sinuous mode; $(b)$ the spanwise fundamental, varicose mode; $(c)$ the spanwise subharmonic, sinuous mode; $(d)$ the spanwise subharmonic, varicose mode. Only the first component of the spanwise Floquet expansion is shown.

and varicose symmetries are illustrated schematically in figure 6 for the spanwise fundamental (figure $6 a, b$ ) and subharmonic (figure $6 c, d$ ) modes.

When the base streaks are steady, all perturbation patterns in the figure exhibit a well-defined and unchanging symmetry. Consider, for example, a steady $u_{1}$ profile, indicated by the solid line on the left. The perturbation streamlines in figures $6(a)$ and $6(c)$ meander in a sinuous motion relative to the low-speed streak at $z=0$. On the other hand, the perturbation patterns in figures $6(b)$ and $6(d)$ are varicose: they have the appearance of a sausage instability relative to the low-speed streak at $z=0$. Since the $u_{1}$ profile was assumed to be steady, these symmetries are preserved at all times.

The case of unsteady base streaks is depicted schematically by the solid and dashed $u_{1}$ profiles, which are separated in time by an interval $\Delta t=\pi / \omega$. The solid profile has the low-speed streak centred at $z=0$, and the dashed profile around $z= \pm 1$. Independent of the streak unsteadiness, the fundamental modes (figure $6 a, b$ ) remain distinct. However, the subharmonic disturbances (figure $6 c, d$ ) become indistinguishable: a sinuous mode relative to the solid $u_{1}$ profile $(z=0)$ becomes varicose at a later time, $t=t_{0}+\pi / \omega$, relative to the dashed $u_{1}$ streak $(z= \pm 1)$, and vice versa. Since the Floquet problem is formally an integration over all phases of the base flow, the spanwise subharmonic eigenmodes from the sinuous and varicose expansions collapse in the unsteady case. This property served as one of the validation criteria for the numerical solution of the eigenvalue problem. 


\subsection{Numerical solution of the eigenvalue problem}

The doubly periodic Floquet problem defined in this section bears a similarity to the work of Liu et al. (2008b), but also introduces additional complexity in the construction of the discrete stability operator. In the work of Liu et al. (2008b), the base flow was a superposition of the Blasius profile and two primary distortions, each periodic in only one dimension: their saturated $\mathrm{T}-\mathrm{S}$ wave was periodic in the $x$-direction and the steady streaks were periodic in the $z$-direction. Therefore, the convolution terms in the secondary stability equations coupled wavenumbers either in $x$ or in $z$, but not in both directions. In the current formulation, the base streaks are periodic in both $t$ and $z$, and therefore convolution terms can couple wavenumbers in both directions. As a result, the numerical algorithm used to solve the eigenvalue problem extends the work of Liu et al. (2008b). A validation of the algorithm is presented in Appendix B, with reference to the literature on stability calculations for time-dependent and spanwise-dependent base flows.

The algorithm is based on a Chebyshev representation of the instability modes in the wall-normal direction. The semi-infinite physical domain is truncated at $y=y_{\infty}$, which was typically 10 boundary-layer thicknesses. The mapping of the GaussLobatto points from the interval $[-1,1]$ to the physical domain $\left[0, y_{\infty}\right]$ clustered the collocation points inside the boundary layer. In order to ensure convergence of the numerical solution, at least 50 Gauss-Lobatto points are required. In addition, at least four Floquet modes are required in the expansion in each of the periodic dimensions. This yielded a system size of the order of 41 million elements. The implicitly restarted Arnoldi iteration method, described by Lehoucq, Sorensen \& Yang (1998), was used for the solution of the eigenvalue problem. The computational cost was reduced by evaluating a subset of the eigenspectrum, which captured the modes with the largest growth rates, $\sigma_{r}$. The two most unstable modes are of particular interest in the current study, and are discussed in detail in the following section.

\section{Linear instability modes of the streaky boundary layer}

In this section, the results of the linear stability analyses are presented for the range of streak parameters discussed in $\S 2.1$. The Reynolds number is $R e=360$, and the streak frequencies and amplitudes span the range, $F \in[0,60]$ and $A_{u} \in$ $[0,20] \%$, respectively. The streak width is approximately $1.8 \delta_{99}$, at the target Reynolds number. These parameters of the base flow are typical of the streaky boundary layer upstream of the inception of turbulent spots in experiments and simulations of bypass transition (Westin et al. 1994; Matsubara \& Alfredsson 2001; Jacobs \& Durbin 2001). The experiments reported root-mean-square streak amplitudes reaching nearly $10 \%$, upstream of transition. It is possible that some instantaneous streaks in the experiments exceeded $A_{u}=20 \%$, as noted by Nolan et al. (2010), which would further promote instability.

First, the stability of boundary layers in the presence of steady streaks, $F=0$, is investigated over a range of streak amplitudes. This problem has been analysed before, for example, by Andersson et al. (2001) in the inviscid limit, and by Cossu \& Brandt (2004). In those papers, the streaks were the boundary-layer response to an optimal primary disturbance, which is a streamwise vortex. Here, the streaks are forced by the free-stream vortical disturbance described in $\S 2.1$.

The growth rate of the most unstable mode is plotted in figure 7 versus streak amplitude. The results show two different modes of instability, and each becomes most unstable at a different streak amplitude. In the absence of streaks, $A_{u}=0$, 


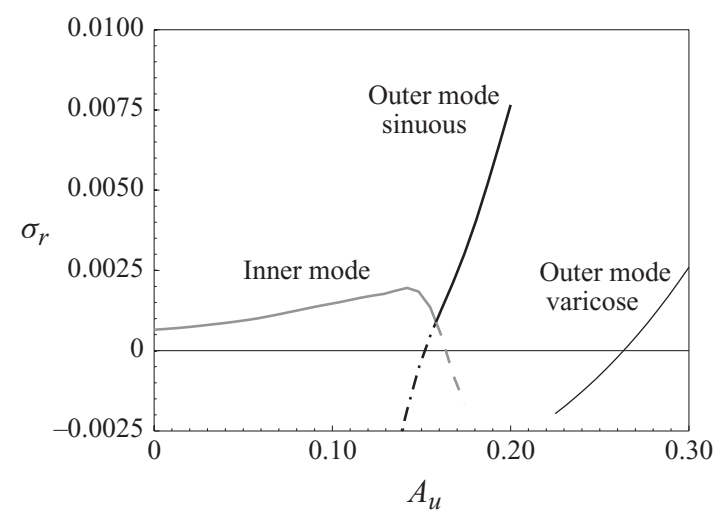

Figure 7. The influence of the amplitude of steady streaks $(F=0)$ on growth rate. Three types of modes are plotted. The grey line $(-)$ is the growth rate of the inner mode; the black line $(-)$ is the outer mode. Both the sinuous and varicose outer modes are shown.
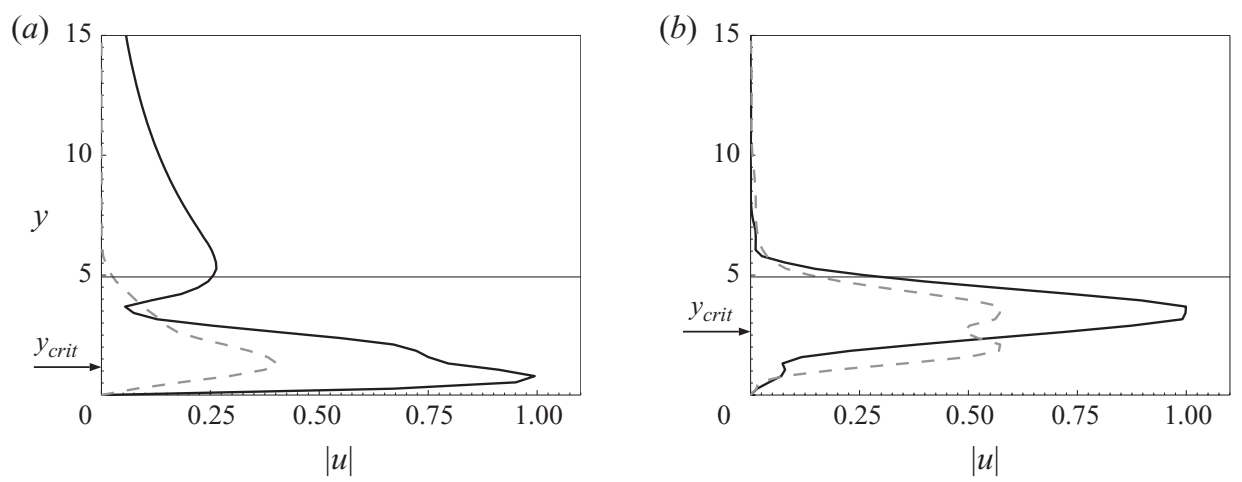

FIGURE 8. Wall-normal profiles of $(a)$ the inner and $(b)$ the outer modes. The absolute value of the $u$-disturbance is plotted for ( - ) the most energetic and (---) the second most energetic components of the Floquet expansion. The location of the critical layer, $y_{\text {crit }}$, is marked by an arrow. The amplitudes of the base streaks are selected to yield unstable modes: $A_{u}=5 \%$ for the inner mode, and $A_{u}=20 \%$ for the outer mode.

the Tollmien-Schlichting wave is the only unstable wave. As $A_{u}$ is increased, the Tollmien-Schlichting wave is slightly destabilized, before the stabilizing influence of the streaks, which was previously described by Cossu \& Brandt (2004) and by Liu et al. (2008b), takes hold. The mode shape for this instability wave is plotted in figure $8(a)$. It is normalized such that the most energetic Floquet component has unit amplitude; the same normalization is adopted for all the eigenfunctions presented herein. The mode shape closely resembles the classical Tollmien-Schlichting wave from Orr-Sommerfeld theory. Its critical layer is near the wall, and therefore will be designated the inner mode.

As the streak amplitude is increased further, the flow becomes unstable to a different type of mode with a high phase speed. Andersson et al. (2001) predicted that a subharmonic, sinuous instability would emerge first, at a streak amplitude of $26 \%$. Indeed, our analysis also predicts that the sinuous mode is the most unstable, however, at a much lower streak amplitude than Andersson et al. (2001). The growth rate of the varicose counterpart is also reported in the figure, and becomes unstable 


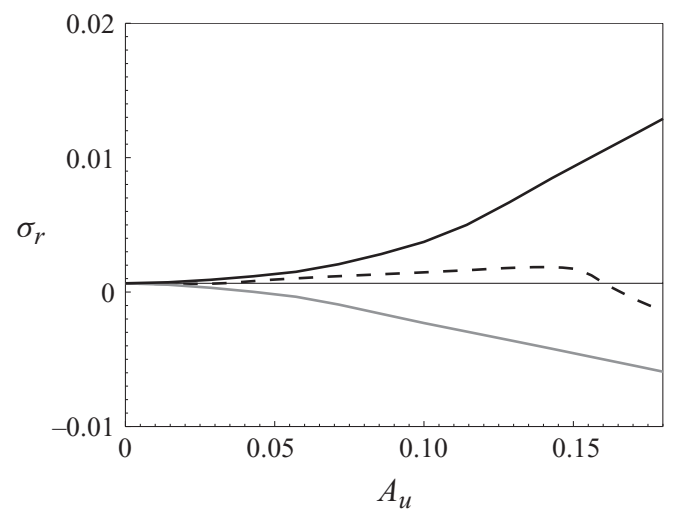

FIGURE 9. The growth rate of the inner mode as a function of streak amplitude for steady streaks $(---)$. The growth rates are also shown for $(-)$ the most stabilizing and ( - ) most destabilizing phases of the streak with $F=20$.

at a higher $A_{u}$. The mode shape of the sinuous instability is plotted in figure $8(b)$. Due to its higher phase speed, it is designated the outer mode.

In bypass transition due to free-stream vortical disturbances, boundary-layer streaks are not steady. Therefore, the influence of unsteadiness in $u_{1}$ on the inner and outer instabilities will be investigated in the following two subsections, respectively. For each mode, the results of two classes of analyses will be considered. First, a quasi-steady approximation of the streaks will be adopted, and the growth rates of the instability will be reported maximized over all possible phases of the base streak. This will be followed by the stability results which take into account the temporal periodicity of $u_{1}$ in the formulation of the eigenvalue problem.

\subsection{The inner mode}

The inner mode exhibits varicose symmetry with respect to the base flow, and is fundamental to the spanwise wavenumber of the base streak. Its Floquet representation is therefore in terms of $\beta_{n}=n k_{z}$, where $k_{z}$ is the streak spanwise wavenumber, and $n$ is an integer multiple. First, a quasi-steady approximation of the base flow is considered, and hence the inner mode has the following form:

$$
\left.\begin{array}{l}
u_{3}=\mathrm{e}^{(\mathrm{i} \alpha x+\sigma t)} \sum_{n=0}^{\infty} u_{n, \cos _{z}} \cos \left(n k_{z} z\right), \\
v_{3}=\mathrm{e}^{(\mathrm{i} \alpha x+\sigma t)} \sum_{n=0}^{\infty} v_{n, \cos _{z}} \cos \left(n k_{z} z\right), \\
w_{3}=\mathrm{e}^{(\mathrm{i} \alpha x+\sigma t)} \sum_{n=1}^{\infty} w_{n, \sin _{z}} \sin \left(n k_{z} z\right) .
\end{array}\right\}
$$

The spanwise independent wavenumber, $\beta_{n}=0$, is part of the above expression, and is composed of only the $u$ and $v$ perturbations; the $w$ component vanishes due to anti-symmetry with respect to the base streak. As the amplitude of the streaks is decreased, $A_{u} \rightarrow 0$, only this two-dimensional component persists and the inner mode reduces to the conventional Tollmien-Schlichting wave of the Blasius boundary layer.

The origin of the inner mode in the classical Tollmien-Schlichting wave is made clear by tracking the growth rate as the amplitude of streaks is smoothly increased from zero. Three curves are contrasted in figure 9: the first is the growth rate for 

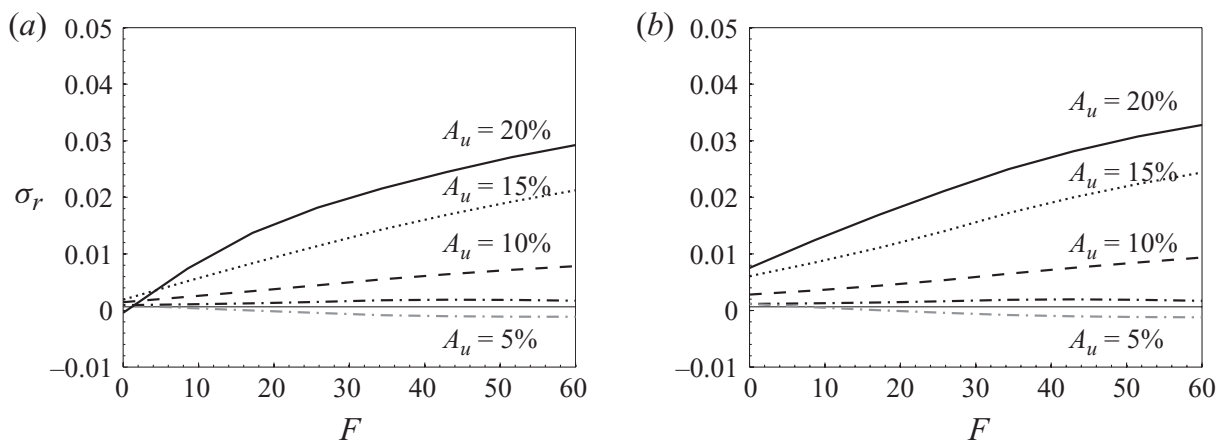

FIGURE 10. The effect of streak amplitude and frequency on the inner mode. The most unstable phase of the unsteady streaks is used in a steady analysis. In $(a)$, the mean flow is modified from Blasius due to nonlinear effects of the streak; in $(b)$ the Blasius profile is used. -.- , $A_{u}=5 \% ;---, A_{u}=10 \% ; \cdots, A_{u}=15 \% ;-, A_{u}=20 \%$. The growth rate for the most stabilizing phase of the base streaks is also shown $(-\cdot-)$ for $A_{u}=5 \%$.

the steady base streak (adopted from figure 7), and the other two curves are for the unsteady base streak with $F=20$. The analysis assumed a quasi-steady base flow, which amounts to 'freezing' a particular phase of the unsteady streak and computing the growth rate. The two curves for $F=20$ represent the most stable and the most unstable phases of the streak profile. In the limit $A_{u}=0$, the streaks vanish and the growth rate of the inner mode exactly matches the Tollmien-Schlichting wave. However, as $A_{u}$ is increased, the behaviour of the inner mode is sensitive to the base flow. In the case of steady streaks, the growth rate initially increases, although inappreciably, followed by a stabilizing effect at higher streak amplitudes, $A_{u} \sim 16 \%$. When one considers the unsteady streak, the growth rate of the most unstable phase steadily increases with $A_{u}$, indicating a significant destabilization of the inner mode. This trend emphasizes the potential importance of streak unsteadiness, where particular phases of the streak profile can lead to enhanced amplification rates of the inner instability. The results are also consistent with the 'intermittent instability' proposed by Wu \& Choudhari (2003). It should be noted, however, that some phases of the base streak have a stabilizing influence. The growth rate at the most stabilized phase is also plotted in figure 9. Whether the 'intermittent instability' or 'intermittent enhanced stability' dominates cannot be inferred from the analysis of frozen base states. However, the analysis highlights the importance of considering the unsteadiness of the base flow in determining the stability of the boundary layer with respect to the inner mode.

The analysis using the quasi-steady base-flow assumption was carried out over a range of streak frequencies, $F \in[0,60]$ and the results are reported in figure $10(a)$. The inner instability is enhanced with increasing $A_{u}$, and this dependence is more pronounced at higher frequencies of the base streak. The influence of the streak unsteadiness only enters through the phase of the base-flow profile, yet causes an appreciable increase in the growth rate of the inner mode. The significant increase in $\sigma_{r}$ is due to the presence of wall-normal inflection points in the base-flow profile, $u_{2}(y ; x, z, t)$. In the steady streak limit, $F=0$, the base flow is not inflectional in $y$ and, as a result, the T-S waves preserve their weak viscous growth rates. However, in the presence of unsteady streaks, wall-normal inflection points were introduced in the base-flow profile. For example, at $F=20$ and $A_{u}=20 \%$, the most unstable temporal phase of the base flow, $u_{2}(y ; x, z, t)$, was inspected for wall-normal inflection points. 

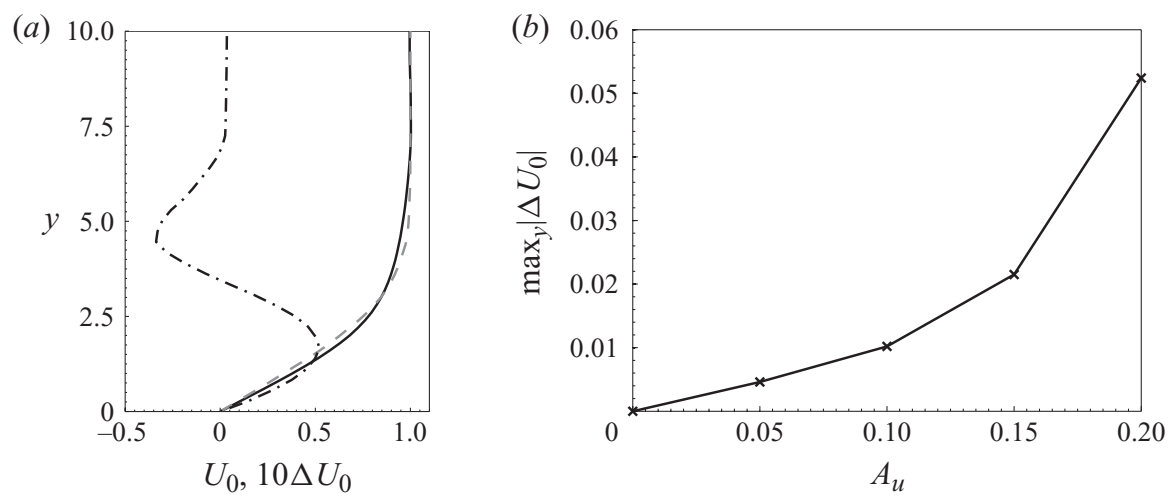

FiguRE 11. (a) Mean flow $U_{0}(-)$ plotted for the case of steady base streaks at amplitude $A_{u}=20 \%$. Also shown is the Blasius solution (---), and the difference between the two profiles premultiplied by $10(-\cdot-)$. (b) Maximum deviation of the mean flow from Blasius, $\max _{y}\left|\Delta U_{0}\right|$ plotted versus streak amplitude at $F=0$.

This exercise was repeated for different $z$-locations, and approximately $60 \%$ of the profiles were inflectional in $y$. This level was increased to $90 \%$ for the higher-frequency base flow, $F=60$. Inflectional wall-normal velocity profiles were also reported by Mandal et al. (2010), based on a PIV study of bypass transition. They argued that the inflectional profiles were important for varicose instabilities, such as the present inner mode.

In order to investigate the significance of the mean flow modification, which is caused by the nonlinear nature of the base streaks, the stability analysis is repeated using a Blasius profile, $U_{0}=U_{\text {Blasius }}$. The growth rates are plotted in figure $10(b)$, and are generally increased relative to the results in figure 10(a). Also note that the stabilization at $F=0$ is no longer observed, and must therefore be linked to the $U_{0}$ profile. The deviation of the mean flow from Blasius is shown in figure 11, when the base streaks are steady, $F=0$. The maximum deviation is $5.24 \%$ when the streak amplitude is highest, $A_{u}=20 \%$. The stabilizing influence of the base flow is in agreement with the work of Cossu \& Brandt (2002). They studied the stability of boundary layers with steady streaks, and found that the stabilizing influence was not preserved if the Blasius flow was used instead of the distorted mean profile.

The above results establish that the inner instability is dependent on the frequency of the Klebanoff streaks. However, the analyses invoked a quasi-steady base-flow assumption, by using particular phases of $u_{1}$. While the growth rates were reported for the most unstable phase, other phases of the base streaks are stabilizing and must be accounted for in determining the overall stability characteristic of the boundary layer. For example, figures $10(a)$ and $10(b)$ include the growth rates of the inner mode for the most stabilizing phase of the base flow, at $A_{u}=5 \%$. In the following section, the stability analysis is performed for the time-dependent base flow, thus fully taking into account the unsteady nature of the streaks.

\subsubsection{The influence of unsteady base flow on the inner mode}

The quasi-steady base-flow assumption was relaxed, and the secondary instability problem was re-evaluated for the time-periodic $u_{2}$. Therefore, the disturbance was expressed in terms of a Fourier expansion in time, as well as in the fundamental 

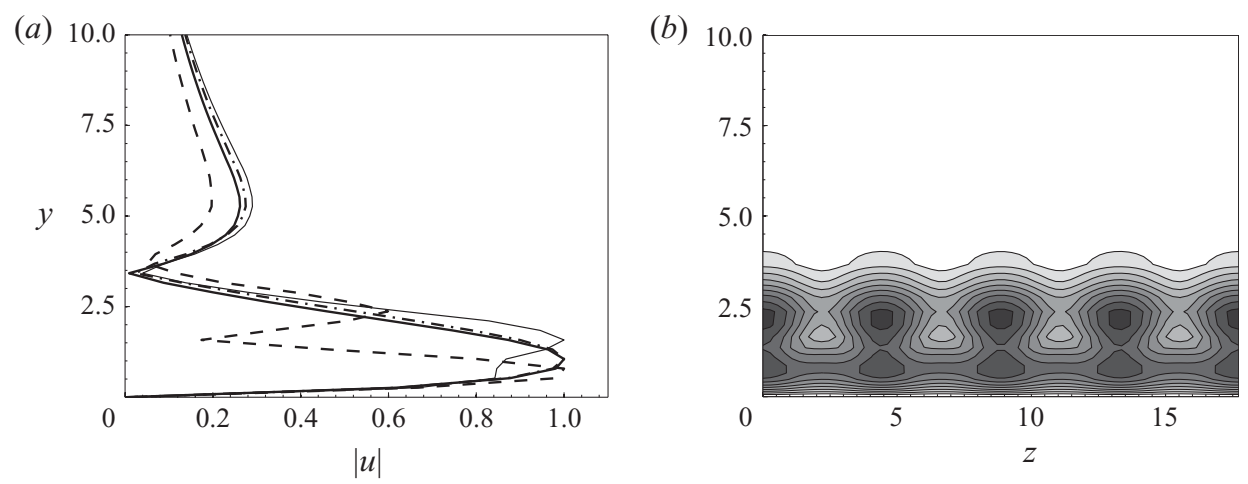

Figure 12. (a) The two-dimensional component in the Floquet expansion of the inner mode. The wall-normal profile of the $|u|$ velocity is plotted for various streak amplitudes, the streak frequency $F=20$ : -,$A_{u}=0.0 \% ;-\cdot-, A_{u}=5.0 \% ;-, A_{u}=7.5 \% ;---, A_{u}=10.0 \%$. $(b)$ The root-mean-square of $u_{3}$ is plotted in the cross-flow plane, for $A_{u}=10.0 \%$. The spanwise domain size is twice the wavelength of the base streak.

spanwise wavenumber of the streaks,

$$
\begin{aligned}
& u_{3}=\operatorname{Re}\left[\mathrm{e}^{(\mathrm{i} \alpha x+\sigma t)} \sum_{m=0}^{\infty} \sum_{n=0}^{\infty}\left(u_{m, n, \cos _{t}, \cos _{z}} \cos (m \omega t)+u_{m, n, \sin _{t}, \cos _{z}} \sin (m \omega t)\right)\right] \cos \left(n k_{z} z\right), \\
& v_{3}=\operatorname{Re}\left[\mathrm{e}^{(\mathrm{i} \alpha x+\sigma t)} \sum_{m=0}^{\infty} \sum_{n=0}^{\infty}\left(v_{m, n, \cos _{t}, \cos _{z}} \cos (m \omega t)+v_{m, n, \sin _{t}, \cos _{z}} \sin (m \omega t)\right)\right] \cos \left(n k_{z} z\right) \text {, } \\
& w_{3}=\operatorname{Re}\left[\mathrm{e}^{(\mathrm{i} \alpha x+\sigma t)} \sum_{m=0}^{\infty} \sum_{n=0}^{\infty}\left(w_{m, n, \cos _{t}, \sin _{z}} \cos (m \omega t)+w_{m, n, \sin _{t}, \sin _{z}} \sin (m \omega t)\right)\right] \sin \left(n k_{z} z\right) .
\end{aligned}
$$

As with the previous steady analyses, the inner mode includes a two-dimensional, or spanwise-independent, Floquet component. The $|u|$-profile for this term is plotted in figure 12(a), for base streak frequency $F=20$. As $A_{u}$ vanishes, this mode collapses onto the classical Tollmien-Schlichting wave. In the presence of streaks, however, the inner mode takes on a three-dimensional form. This is shown in figure 12(b), where $u_{3}$ is plotted in the cross-flow plane. The domain includes two spanwise wavelengths of the streak.

The effect of base-flow unsteadiness on the inner instability is shown in figure 13, where the growth rate is plotted versus streak frequency, at different streak amplitudes. The results account for all phases of the base streak, both stabilizing and destabilizing. The inner mode is generally destabilized by high-amplitude, unsteady streaks, and its growth rate significantly surpasses the viscous Tollmien-Schlichting wave.

A comparison of the present results to the previous quasi-steady calculations in figure 10 illustrates the importance of accounting for all phases of the unsteady base streak. Consider, for example, the high-frequency streak, $F=60$. At high amplitude, both analyses predict a destabilization of the inner mode. The temporal Floquet expansion, however, predicts a larger growth rate than the most unstable phase in the quasi-steady results. Furthermore, at low amplitude, the temporal Floquet expansion predicts a net stabilizing effect, which could not be inferred from the quasi-steady computations. 


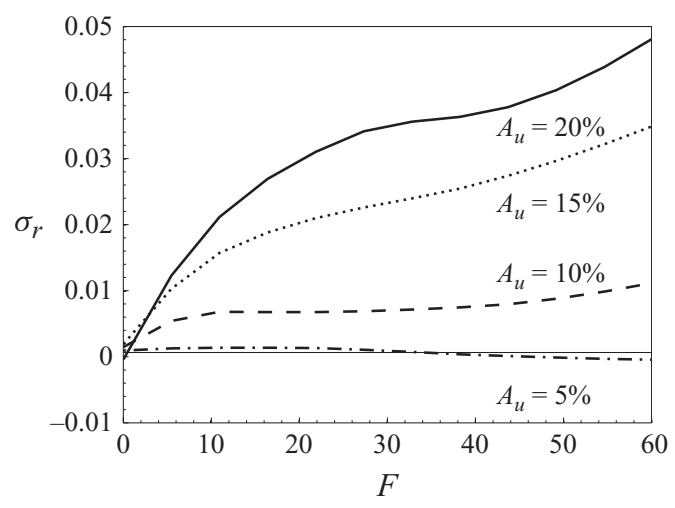

FigurE 13. The influence of streak amplitude and frequency on the inner mode. -.- , $A_{u}=5 \% ;---, A_{u}=10 \% ; \cdots, A_{u}=15 \% ;-, A_{u}=20 \%$.

(a)

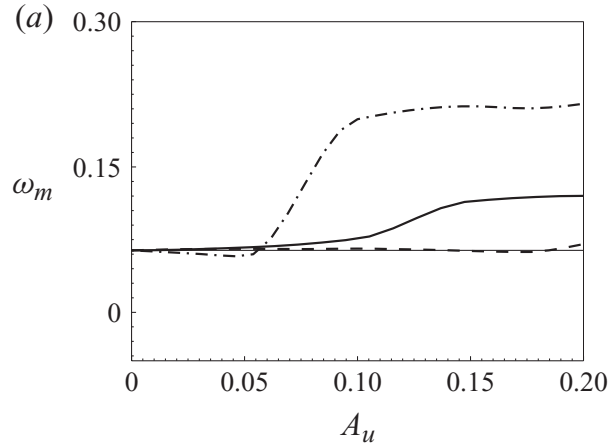

(b)

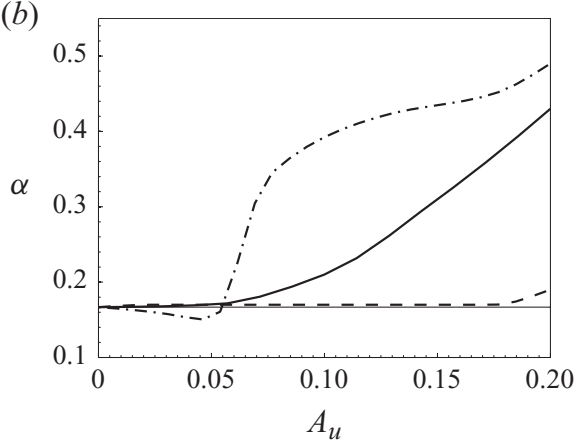

FiguRE 14. Effect of $A_{u}$ on $(a)$ the frequency of the most energetic component, and $(b)$ the streamwise wavenumber of the inner mode. The base streak frequencies are:,$--- F=0 ;-$ $F=20 ;-\cdot-, F=60$.

According to the results of figure 13, the inner mode can be stabilized, albeit inappreciably, in two regimes: (i) steady, high-amplitude streaks which cause a stabilizing modification to the mean flow, and (ii) low-amplitude, higher-frequency streaks. The enhanced stability for high-frequency, low-amplitude streaks can be compared to the work of Grosch \& Salwen (1968). They studied the stability of timedependent plane Poiseuille flow. Their base flow included a steady and an unsteady component - akin to the present mean flow with unsteady streaks. They found that modest amplitudes of the unsteady component of the base flow tended to stabilize the flow, whereas the higher amplitudes made the flow unstable. Similar observation can be made in the current context. The stabilization effect is, however, not significant in comparison to the substantial increase in growth rates due to unsteady streaks with amplitude $A_{u}>10 \%$.

The most energetic component of the Floquet expansion (3.2) was identified, and its frequency $\omega_{m}=m \omega+\sigma_{i}$ and streamwise wavenumber $\alpha$ are shown in figures $14(a)$ and $14(b)$ as a function of $A_{u}$. Both quantities are insensitive to the amplitude of the steady base streaks. However, unsteady streaks with amplitude $A_{u}>6 \%$ cause both the frequency and wavenumber of the inner mode to increase, and the effect increases with streak frequency. The results of the linear theory are in agreement with Kendall (1991) who observed instabilities similar to the Tollmien-Schlichting waves, except 

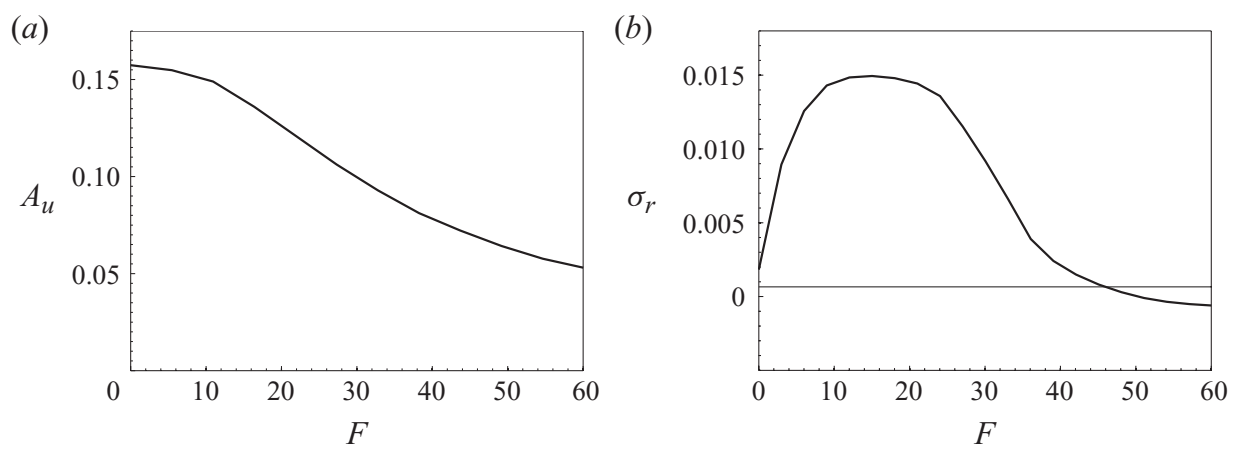

Figure 15. (a) The dependence of streak amplitude $A_{u}$ on $F$, when $A_{v}=4.5 \times 10^{-3}$ is held constant. (b) Growth rate of the inner mode versus the streak frequency at constant $A_{v}$. The growth rate of the $\mathrm{T}-\mathrm{S}$ wave for the Blasius boundary layer is marked by the horizontal line.

that their frequency and growth rate were higher in the presence of Klebanoff streaks. According to the current results, the phase speed of the inner mode also exceeds that of the Tollmien-Schlichting waves, $c_{r}=0.38$. The maximum computed phase speed was $c_{r}=0.54$, over the range $A_{u}$ and $F$ considered.

The discussion of the inner instability has thus far overlooked the propensity of lower-frequency streaks to reach higher amplitudes, $A_{u}$. In particular, for the same level of free-stream forcing, lower-frequency vortical disturbances penetrate deeper into the boundary layer, and cause amplification of the Klebanoff streaks to higher $A_{u}$ levels (Zaki \& Durbin 2005). It is therefore more appropriate to consider constant values of $A_{v}$ or $A_{w}$ of the free-stream vortical mode which causes the generation of streaks. The strength of the streak response, $A_{u}$, is frequency-dependent, and is determined from DNS.

The value of $A_{v}$ was selected such that the induced streaks reached amplitudes $A_{u}$ of the order of $15 \%$ in the low-frequency limit. The dependence of $A_{u}$ on frequency is shown in figure $15(a)$. In figure $15(b)$, the growth rate of the inner instability is plotted versus streak frequency, here at constant $A_{v}$. In the steady streak limit, $F=0$, the inner mode is only slightly unstable due to the modification to the Blasius profile, despite the high value of $A_{u}$. The growth rate increases rapidly as the streaks become unsteady, especially in the lower frequency range where they reach high amplitude. While higher levels of unsteadiness are destabilizing, the streak amplitude $A_{u}$ decreases with frequency and reduces the instability growth rate. Therefore, an optimal range of streak frequencies emerges. Lower-frequency streaks do not introduce sufficient unsteadiness, and higher-frequency Klebanoff modes occur at weaker, more stable amplitudes.

In the context of bypass transition, a spectrum of streak amplitudes and frequencies is generated in response to forcing by free-stream turbulence. The low-frequency components of this forcing, not $F=0$, play the most significant role as regards enhancing the inner instability. The high-frequency components are less significant due to their low propensity to generate high-amplitude streaks.

\subsection{The outer mode}

The existence of the outer mode is due entirely to streaks. Unlike the inner instability which reduces to a Tollmien-Schlichting wave, the outer mode ceases to exist when the amplitude of the streak $A_{u}$ is set to zero. Its phase speed is approximately $c_{r} \sim 0.75 U_{\infty}$, which is in agreement with previous experimental and numerical studies of streak 

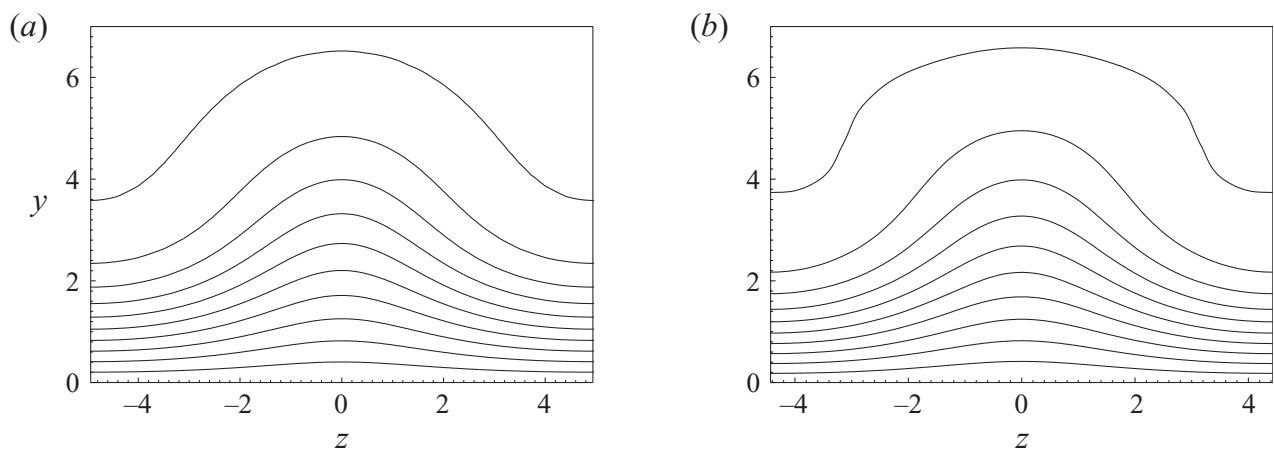

FigURE 16. Contours of the streamwise velocity $u_{2}(y, z)=U_{0}(y)+u_{1}(y, z)$ for steady streaks at amplitude $A_{u}=20 \%$. The contour levels correspond to $0<u_{2}<1$ with an increment of 0.1. (a) The base flow profile kindly provided by Dr L. Brandt. It is obtained from DNS of the linearly optimal streaks, at the location of maximum streak amplitude (Andersson et al. 2001). (b) The streaks are due to free-stream vortical forcing, and are extracted at $R e=360$, $k_{y}=0.677, k_{z}=0.71$, based on the local Blasius length scale.
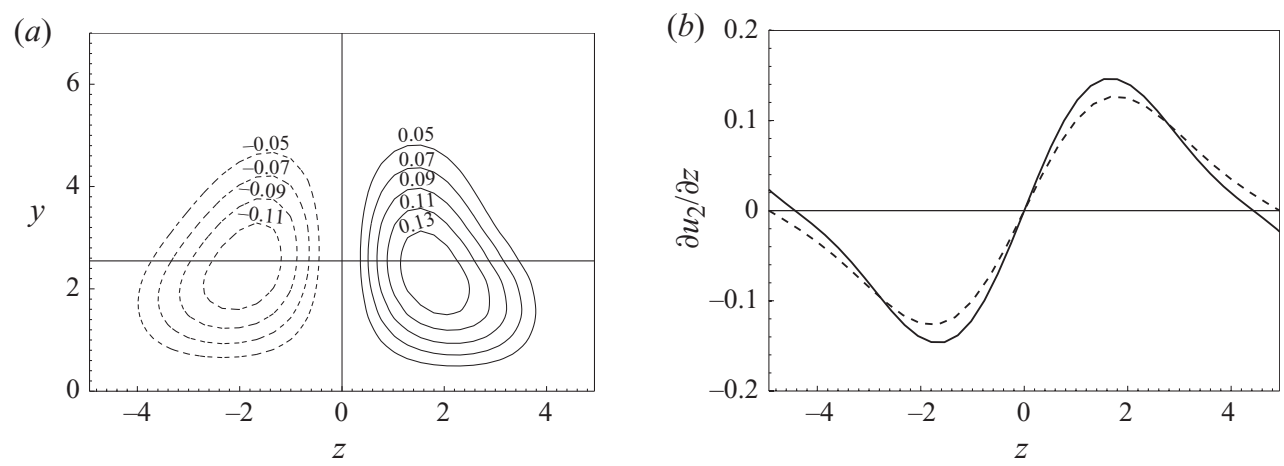

FIGURE 17. (a) Contours of the spanwise shear for the velocity fields in figure 16. The left half of the contour plot corresponds to the streaks by Andersson et al. (2001). The right half corresponds to the streaks forced by free-stream vortical modes. The horizontal line marks the critical layer of the outer mode. (b) The spanwise shear at the critical layer. ---, streaks by Andersson et al. (2001); _ , streaks forced by free-stream vortical modes.

instability. For example, the experiments of Mans et al. (2007) and Swearingen \& Blackwelder (1987), as well as the DNS of Jacobs \& Durbin (2001), all reported an instability phase speed which was closer to the free-stream velocity than the conventional Tollmien-Schlichting wave.

Given the origin of the outer instability in the streaks, it is natural to seek a comparison to the findings of Andersson et al. (2001). Their inviscid analysis predicts that the subharmonic, sinuous instability emerges first, at a critical streak amplitude of $26 \%$, followed by the varicose mode at $37 \%$. Our results for the steady streak $F=0$ were shown in figure 7 . Indeed the subharmonic, sinuous mode is most unstable. However, its critical amplitude, $A_{u}=15.2 \%$, is much lower than the threshold predicted by Andersson et al. (2001).

While our predicted threshold for instability is closer to the experimentally observed level, it remains important to explain the disagreement with Andersson et al. (2001). In that paper, the streaks were computed using DNS, as is the case herein. The main difference arises in the model of the base flow. Andersson et al. (2001) studied the 

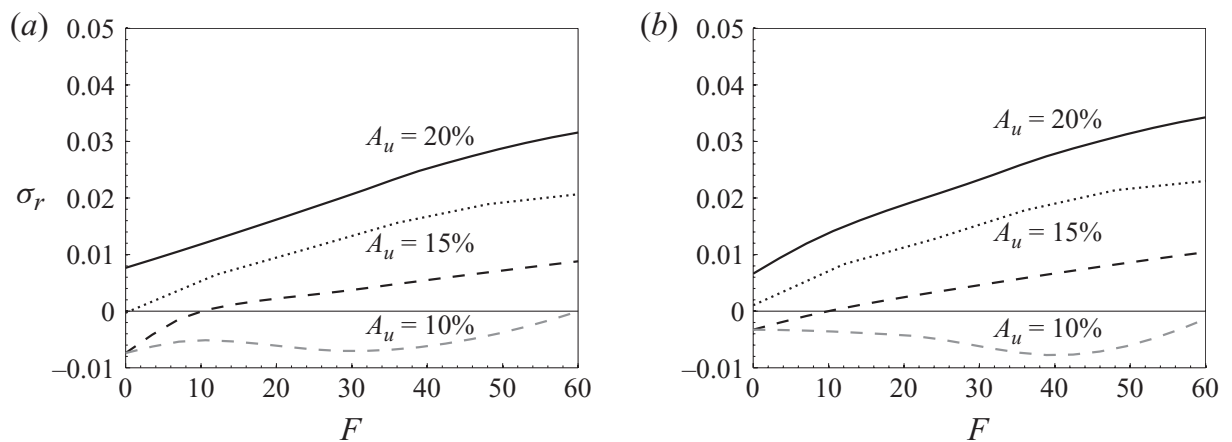

FIGURE 18. The effect of streak amplitude and frequency on the outer mode. The most unstable phase of the unsteady streaks is used in a steady analysis. In $(a)$, the mean flow is modified from Blasius due to nonlinear effects of the streak; in $(b)$ the Blasius profile is used. $A_{u}=20 \% ; \cdots, A_{u}=15 \% ;---, A_{u}=10 \%$. The growth rate for the most stabilizing phase of the base streaks is also shown (---) for $A_{u}=10 \%$.

stability of the 'optimal' streak which was generated by a streamwise vortex within the boundary layer. Our streaks, however, are forced by free-stream vortical disturbances representative of the influence of far-field turbulence on boundary layers in bypass transition. The base flow used in the stability analysis of Andersson et al. (2001) was kindly provided by Dr L. Brandt. Contours of their $u_{2}$ are plotted in figure 16(a) for the case where the streak amplitude is $A=20.2 \%$. The corresponding base flow from the current analysis, for a steady streak with amplitude $A_{u}=20 \%$, is shown in figure 16(b). Despite the similarity, the spanwise variation in the base flow is less pronounced in the case of optimal streaks. As a result, the spanwise shear associated with the base streaks considered herein is noticeably higher, for the same streak amplitude (see figure 17). Since the secondary instability is related to the spanwise shear, it is natural that the critical amplitude in the current work is lower than that obtained by Andersson et al. (2001).

Similar to the discussion of the inner instability, the outer mode will be first investigated in the context of a quasi-steady base flow. This approximation is subsequently relaxed, and the time periodicity of the base flow is incorporated in the analysis.

The outer instability is subharmonic in the spanwise wavenumber of the streaks, and exhibits sinuous symmetry in that dimension. Therefore, for a frozen base flow, the outer mode can be expressed according to

$$
\left.\begin{array}{l}
u_{3}=\mathrm{e}^{(\mathrm{i} \alpha x+\sigma t)} \sum_{n=0}^{\infty} u_{n, \cos _{z}} \cos \left(\left[\frac{2 n+1}{2}\right] k_{z} z\right), \\
v_{3}=\mathrm{e}^{(\mathrm{i} \alpha x+\sigma t)} \sum_{n=0}^{\infty} v_{n, \cos _{z}} \cos \left(\left[\frac{2 n+1}{2}\right] k_{z} z\right), \\
w_{3}=\mathrm{e}^{(\mathrm{i} \alpha x+\sigma t)} \sum_{n=0}^{\infty} w_{n, \sin _{z}} \sin \left(\left[\frac{2 n+1}{2}\right] k_{z} z\right) \cdot
\end{array}\right\}
$$

The above expansion is substituted in the stability equations, (2.6)-(2.8), and the eigenvalue problem is solved for the growth rate $\sigma_{r}$. All phases of the base streaks were considered, over a range of streak frequencies and amplitudes. The growth rate of the outer instability is reported, for the most unstable phase, in figure 18(a). The outer mode becomes increasingly more unstable in the presence of high-amplitude 
streaks. Also, increasing the frequency of the base streak has a destabilizing effect. Results for the most stable phase of the base flow are also plotted for streak amplitude, $A_{u}=10 \%$. The analysis was also repeated using the Blasius profile, in place of the distorted mean flow, and the results are shown in figure 18(b). A comparison of figures $18(a)$ and $18(b)$ indicates that the effect of the modification to the Blasius profile by the nonlinear streaks is small. This is in agreement with the view that streak instability is due to spanwise shear between adjacent streaks (see, for example, Swearingen \& Blackwelder 1987), and is therefore insensitive to the modification of the mean flow.

The preceding results confirm that some phases of unsteady boundary-layer streaks can be destabilizing, and lead to the amplification of the outer mode. However, the quasi-steady approximation of the base flow does not account for other phases of the streak, which can have a stabilizing effect. In the following subsection, the time-periodicity of the unsteady streaks is retained in the stability analysis.

\subsubsection{The influence of unsteady base flow on the outer mode}

Since the outer mode is subharmonic relative to the spanwise wavenumber of the streak, a classification into sinuous and varicose is not required; both the sinuous and varicose eigenmodes coalesce as explained in $\S 2.2$. The Fourier representation of the outer mode is given below. When substituted in the stability equations (2.6)-(2.8), it yields the eigenvalue problem for the complex frequency, $\sigma$,

$$
\begin{aligned}
& u_{3}=\mathrm{e}^{(\mathrm{i} \alpha x+\sigma t)} \sum_{m=0}^{\infty} \sum_{n=0}^{\infty}\left(u_{m, n, \cos _{t}, \cos _{z}} \cos (m \omega t)\right. \\
& \left.+u_{m, n, \sin _{t}, \cos _{z}} \sin (m \omega t)\right) \cos \left(\left[\frac{2 n+1}{2}\right] k_{z} z\right), \\
& v_{3}=\mathrm{e}^{(\mathrm{i} \alpha x+\sigma t)} \sum_{m=0}^{\infty} \sum_{n=0}^{\infty}\left(v_{m, n, \cos _{t}, \cos _{z}} \cos (m \omega t)\right. \\
& \left.+v_{m, n, \sin _{t}, \cos _{z}} \sin (m \omega t)\right) \cos \left(\left[\frac{2 n+1}{2}\right] k_{z} z\right), \\
& w_{3}=\mathrm{e}^{(\mathrm{i} \alpha x+\sigma t)} \sum_{m=0}^{\infty} \sum_{n=0}^{\infty}\left(w_{m, n, \cos _{t}, \sin _{z}} \cos (m \omega t)\right. \\
& \left.+w_{m, n, \sin _{t}, \sin _{z}} \sin (m \omega t)\right) \sin \left(\left[\frac{2 n+1}{2}\right] k_{z} z\right) .
\end{aligned}
$$

The two most energetic components of the above expansion are plotted in figure 19(a) for representative streak parameters. Figure 19(b) shows contours of $u_{3}$ in the cross-stream plane. The size of the spanwise region shown is twice the wavelength of the base streak. Therefore, the figure contains only one wavelength of the subharmonic, outer mode. Compared to the inner mode, much of the energy is removed from the wall, and the critical layer is in the outer region, close to the free stream. The most unstable streamwise wavenumber was around $\alpha=0.3$, which agrees closely with the instability predicted by Andersson et al. (2001). The corresponding wavelength is of the same order as the streak instabilities observed in the DNS of Brandt et al. (2004), where $\lambda_{x} \sim 5 \delta^{*}$.

The growth rate of the outer mode is plotted versus the frequency of the base streak, at various levels of $A_{u}$ in figure 20(a). The analysis, which takes into account all phases of the base-flow unsteadiness, demonstrates that the outer mode is destabilized by high-amplitude, unsteady streaks. When the streaks are weak, $A_{u}=5 \%$, a stabilizing effect is observed at higher frequencies, similar to the inner mode. 

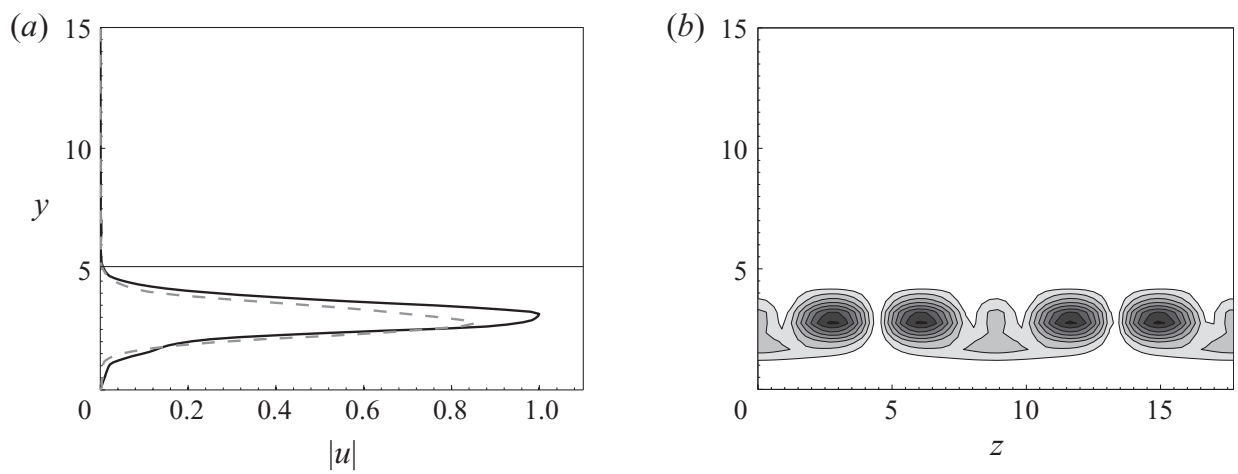

FIGURE 19. (a) The wall-normal profile for the outer mode in the presence of unsteady streaks. The absolute value of the $u$-disturbance is plotted for $(-)$ the most energetic $\left(\omega_{m}=0.2754\right.$, $\left.\beta_{n}=1.0635\right)$, and $(---)$ second most energetic $\left(\omega_{m}=0.2682, \beta_{n}=1.0635\right)$ components of the Floquet expansion. The parameters of the base streak are $A_{u}=10 \%, F=20$. (b) The root-mean-square of $u_{3}$ is plotted in the cross-flow plane. The spanwise domain size is twice the wavelength of the base streak.
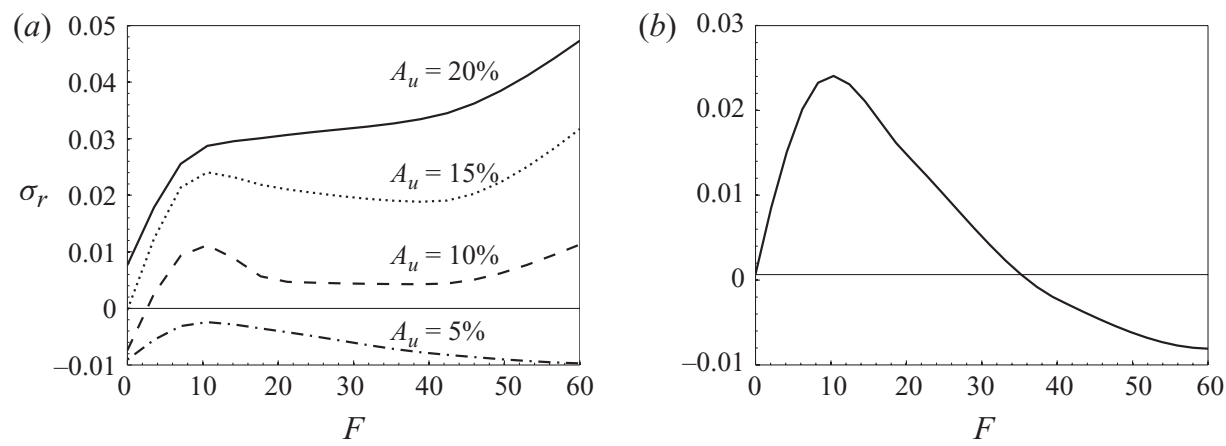

Figure 20. The effect of streak frequency on the growth rate of the outer mode. The streak amplitudes, $A_{u}$, are kept constant in $(a)$, and $(b)$ shows results at constant $A_{v}$.

As indicated in the discussion of the inner mode, it is not sufficient to evaluate the growth rate of the outer instability at constant $A_{u}$. Instead, the propensity of lowfrequency streaks to reach higher amplitude must be taken into account. Therefore, the amplitude $A_{v}$ of the primary disturbance was held fixed (the corresponding $A_{u}\left(F ; A_{v}\right)$ was reported in figure $\left.15 a\right)$, and the growth rate of the outer instability was re-evaluated. The results, shown in figure $20(b)$, indicate the presence of an optimal range of streak frequencies. Streaks with lower wavenumbers can reach high amplitudes, but do not introduce sufficient unsteadiness to enhance the growth rate of the outer instability. Streaks with higher wavenumbers provide that unsteadiness, but do not amplify considerably; their small $A_{u}$ is therefore insufficient to trigger the outer instability. As a result, the most unstable streak frequency, or the 'optimal' streak in the sense of promoting secondary instabilities, emerges near $F \sim 10$.

The critical streak amplitude, $A_{u}^{c}$, for the onset of outer instability is reported in figure 21. The results show that $A_{u}^{c}$ initially decreases as the streak frequency is increased. The predicted critical amplitude compares more favourably with the previously cited experimental observations than the steady, inviscid results of Andersson et al. (2001). For example, even the highest-amplitude streaks in the 


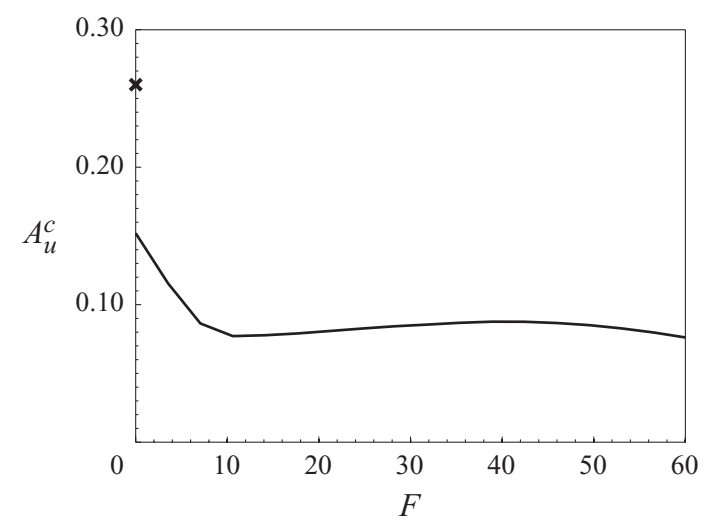

FIGURE 21. Dependence of the critical streak amplitude for the outer instability on $F$. The cross marks the critical value from the work of Andersson et al. (2001).

experiments by Hernon et al. (2007b; see their figure 3, $\left.T_{u}=1.3 \%\right)$ are stable according to the stability criterion by Andersson et al. (2001), but are unstable according to the current results. Therefore, the high instantaneous streak amplitudes relative to r.m.s. values (Hernon, Walsh \& McEligot 2007a; Hernon et al. 2007b), together with the lower stability threshold demonstrated herein, can explain breakdown of streaks in bypass transition, even at moderate free-stream turbulence intensities.

Another notable observation relates to the dominance of sinuous versus varicose instability. Both our steady analyses and the work of Andersson et al. (2001) predict a dominant sinuous mode. On the other hand, Brandt \& Henningson (2002) asserted that the boundary layer had nearly equal propensity to undergo sinuous or varicose instability based on their DNS of bypass transition. This difference can be explained by the results of the unsteady analysis. Due to the spanwise subharmonic nature of the outer mode, it encompasses perturbation patterns that can appear either sinuous or varicose, depending on the local phase of the base streak.

\subsection{Mode competition between the inner and outer instabilities}

In the preceding subsections, the effect of unsteady streaks was discussed with respect to the inner and outer modes independently. It is of interest to compare the growth rates of these two instabilities. Such comparison was presented for the steady base streaks, $F=0$, in figure 7: the inner mode was the only instability at low streak amplitudes, but the outer mode became dominant at higher levels of $A_{u}$. A similar trend is observed when the base streak is of low frequency, for example, at $F=10$, which is shown in figure 22(a). The change in the dominant instability hints at the bypass mechanism in boundary-layer transition. The inner instability (grey curve), which has its origin in the viscous Tollmien-Schlichting wave, is initially the only instability mode but is weakly amplifying. However, as the Klebanoff streaks exceed $A_{u} \sim 7.5 \%$, the inner mode is bypassed by the strongly amplifying outer instability (black curve), whose exponential growth rate $\sigma_{r}$ continues to increase with streak intensity.

The inner-outer mode competition is further examined over a range of frequencies of the base streak in figure 22(b), which provides a good summary. The thin horizontal line in the figure corresponds to the growth rate of the classical Tollmien-Schlichting wave in the two-dimensional Blasius flow. Therefore, the boundary-layer instability is clearly promoted by the presence of high-amplitude, unsteady streaks. In the 


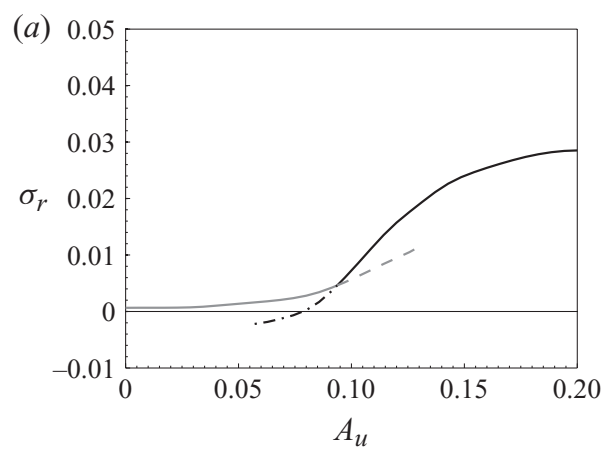

(b)

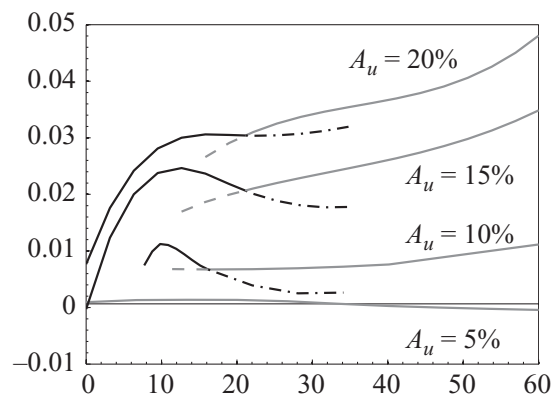

FIGURE 22. The influence of unsteady streaks on the growth rate of the most unstable mode. (a) The growth rate is plotted versus $A_{u}$ for a base streak with $F=10$. (grey line), inner mode; - (black line), outer mode. $(b)$ The growth rate is plotted versus streak frequency, at streak amplitudes $A_{u}=\{0,5,10,15,20\} \%$.

low-frequency regime, the dominance of the outer mode is seen beyond $A_{u} \simeq 10 \%$. It is also interesting to note the dominance of the inner instability at relatively high frequencies of the base streak. This can be due to (i) enhanced parametric resonance between the higher-frequency unsteady streaks and the classical instability waves of the Blasius profile $(137<F<240)$ at the target Reynolds number or (ii) an increase in the number of inflectional profiles of the base flow at higher frequencies of streak modulation.

Based on the above results, the dominant mode is determined by both the streak amplitude and streamwise wavenumber. In bypass transition, the boundary layer contains a spectrum of streaks with various amplitudes and frequencies. It is therefore difficult to predict which secondary instability will dominate. Breakdown can be initiated due to the inner or outer instability, dependent on the flow conditions. Indeed, previous simulations have described secondary instabilities that can be related to the inner and outer types.

\section{Direct numerical simulations}

This section reports on DNS of the inner and outer instabilities in a streaky, zeropressure-gradient boundary layer. The objective is to provide an empirical view of the evolution of these instabilities and their breakdown to turbulence, in a canonical setting. The three-dimensional, incompressible Navier-Stokes equations are solved using the numerical algorithm described in Rosenfeld, Kwak \& Vinokur (1991). The method is a fractional step formulation where the convective terms are treated explicitly using Adams-Bashforth time advancement; the diffusion and pressure terms are advanced by Crank-Nicolson and implicit Euler schemes, respectively. The spatial discretization is based on local volume fluxes on a staggered computational grid.

The inlet boundary layer $99 \%$ thickness, $\delta_{99}^{0}=\delta_{99}\left(x_{0}\right)$, is used as the characteristic length scale in this section, because the Blasius length scale is only meaningful in the laminar region and the DNS domain extends beyond transition into the turbulent region. Using this scaling, the size of the computational domain is $460 \times 20 \times 24$, in the streamwise, wall-normal and spanwise directions, respectively. In terms of Reynolds number, the computational domain spans $163 \leqslant R e \leqslant 628$. The inflow plane is therefore at the same location as the simulations in Jacobs \& Durbin (2001). It is upstream of the critical Reynolds number for the first unstable Tollmien-Schlichting 
wave. While the exit plane is downstream of the critical $R e$, it is well upstream of the location where the orderly transition process would be complete, $R e \approx 1730$.

The number of grid points in the streamwise, wall-normal and spanwise directions are $2049 \times 129 \times 129$. The grid is uniform in the streamwise and spanwise coordinates, but is stretched in the wall-normal direction. The resolution is similar to the DNS by Jacobs \& Durbin (2001), who performed an extensive grid refinement study. Based on wall units, the maximum grid spacings are $\Delta x^{+}=8.1, \Delta y_{w}^{+}=0.4$ and $\Delta z^{+}=6.8$, where $\Delta y_{w}^{+}$is at the wall.

A free-slip condition is applied on the top boundary of the computational domain. The shape of this boundary is determined based on the downstream development of the boundary-layer displacement thickness, in order to ensure zero-pressure-gradient flow. A no-slip boundary condition is enforced at the bottom wall, and a convective outflow condition is applied at the exit plane. At the inlet to the simulation domain, in addition to the Blasius profile, two perturbations are prescribed. The first is the same disturbance as in $\S 2.1$, i.e. a low-frequency, free-stream vortical mode which, downstream of the inlet, leads to the generation of a high-amplitude streaky response inside the boundary layer. Therefore, the boundary-layer streaks in the current simulations are the same as those adopted in our stability analyses. The second disturbance is an inner or outer instability mode obtained from the Floquet analysis of $\S 3$.

The inflow condition is therefore

$$
\begin{aligned}
\boldsymbol{v}_{0}(y, z, t)=\boldsymbol{v}_{B}(y) & +A_{v} \operatorname{Re}\left\{u_{1}\left(y, x_{0}\right) \cos \left(k_{z} z\right) \mathrm{e}^{\mathrm{i}(-\omega t)}\right\} \boldsymbol{e}_{x} \\
& +A_{v} \operatorname{Re}\left\{v_{1}\left(y, x_{0}\right) \cos \left(k_{z} z\right) \mathrm{e}^{\mathrm{i}(-\omega t)}\right\} \boldsymbol{e}_{y} \\
& -A_{v} \operatorname{Im}\left\{w_{1}\left(y, x_{0}\right) \sin \left(k_{z} z\right) \mathrm{e}^{\mathrm{i}(-\omega t)}\right\} \boldsymbol{e}_{z} \\
& +A_{s} \sum_{n} \sum_{m} \operatorname{Re}\left\{\boldsymbol{v}_{n, m}(y) \mathrm{e}^{\mathrm{i}\left(\left[n k_{z}+\gamma\right] z+\left[m \omega+\sigma_{i}\right] t\right)}\right\} .
\end{aligned}
$$

Based on the results of linear theory ( $\$ 3.3$, figure 22$)$, the outer mode dominates for relatively low streak frequencies, and the inner instability is most pronounced at the higher frequencies. Therefore, streaks with frequency $F=30$ were used in the simulations of the outer mode, and $F=60$ for the inner mode. The spanwise wavenumber of the streaks remained unchanged, $k_{z}=0.71$, based on the Blasius length scale at the target Reynolds number $\left(k_{z} \delta_{99}^{0}=1.57\right)$. The value of $A_{v}$ was adjusted such that the root-mean-square streak disturbance was $10 \%$ at the target Reynolds number.

The current simulations of modal interactions bear a similarity to the DNS of Zaki \& Durbin $(2005,2006)$. They simulated pairs of continuous Orr-Sommerfeld modes: a low-frequency and a high-frequency pair. The low-frequency disturbance leads to the generation of streaks. The high-frequency pair did not penetrate the boundary layer due to shear sheltering. Instead, it was in the free stream and interacted with the lifted streaks to cause breakdown to turbulence. It is therefore possible that the instability mechanism in those simulations is related to an 'outer' mode. However, the high-frequency component in that work was not, formally, an instability of the base flow in the sense of linear stability analysis.

\subsection{The inner mode}

The interaction of the inner instability mode with the streaky boundary layer leads to transition to turbulence in our simulations. A top view of the $u$-perturbation field inside the boundary layer is shown in figure 23 . The figure shows the upstream laminar 


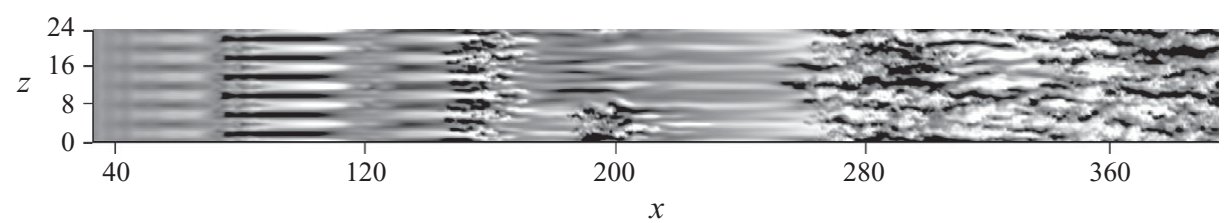

FIGURE 23. Contours of the $u$-perturbation velocity $(-0.10 \leqslant u \leqslant 0.10)$ inside the boundary layer. The plane is $y=0.6$ based on the boundary-layer thickness at the inlet plane. Further downstream, $y / \delta_{99}=0.1$, where $\delta_{99}$ is the boundary-layer thickness at $x=360$.

flow, a turbulent patch in the transition region and the fully turbulent boundary layer near the end of the domain. Turbulent spots first appear near $x \approx 150(R e=383)$, which is slightly downstream of the target Reynolds number for our stability analyses. This suggests that the secondary instability of the streaks took place upstream, near the target Reynolds number, followed by spot inception and breakdown.

Unlike the random forcing of the streaks by free-stream turbulence in bypass transition, the inlet forcing in our controlled simulations is via the inner mode. The inner instability is fundamental to the spanwise wavenumber of the streaks $\beta_{n}=n k_{z}$ (see (3.2)). Therefore, the amplification of the secondary instability initially takes on a repeated pattern in the spanwise direction. As a result, breakdown can be repeated across the span, as seen near $x=160$ in figure 23. However, irregular patterns of breakdown are also observed due to the nonlinear nature of transition to turbulence, which can disrupt the spanwise homogeneity of the flow as seen near $x=200$.

The events which precede the flow field in figure 23 are presented in figures 24 and 25. Each sequence spans the time period $T-100<t<T-20$, where $T$ is the time of the final field in figure 23. The top views show contours of the $u$ - and $w$-perturbation velocities, respectively. They capture the amplification of the innermode instability and nonlinear breakdown to turbulence. Near the inlet (not shown), the inner instability wave is traversed by many streaks due to their relative phase speeds. However, when the instability reaches high amplitude, it is seen to amplify in the region near the overlap of the forward and backward streaks. The subdomains plotted in figures 24 and 25 translate in the downstream direction at $0.625 U_{\infty}$. The inner instability retains the same relative position in the viewing window. Therefore, the phase speed of the inner instability at this stage of breakdown is in reasonable agreement with our Floquet results in $\S 3.1$, but slightly exceeds the propagation speed of the wave packets observed by Nagarajan et al. (2007), $c_{r}=0.52-0.60 U_{\infty}$. The analysis of Goldstein \& Sescu (2008) under-predicted this quantity, and the discrepancy was attributed to the two-dimensionality of their result. The current DNS supports their explanation: the three-dimensionality of the inner instability is evident in the third and fourth frames of figure 25, in the contours of $w$-perturbations. The inclined checkered pattern resembles the spanwise velocity contours reported by Nagarajan et al. (2007), in their figure 13(c).

Iso-surfaces of the $\lambda_{2}$ vortex identification criterion are shown at $t=T-60$, in the middle frames of figures 24 and 25. Near $x=120, \Lambda$-shaped structures are observed at the intersection of the high- and low-speed streaks. They are narrow in the span and short in the streamwise direction, in comparison to the secondary instability of classical T-S waves (Herbert 1988). The $\Lambda$ s are also distinct from those which emerge when two-dimensional T-S waves are modulated by streaks (Liu et al. 2008a). It was shown in $\S 3.1$ that the inner mode reduces to the $\mathrm{T}-\mathrm{S}$ wave when $u_{1}$ vanishes. However, when $u_{1}$ is finite and unsteady, the Floquet analysis predicts that the inner 

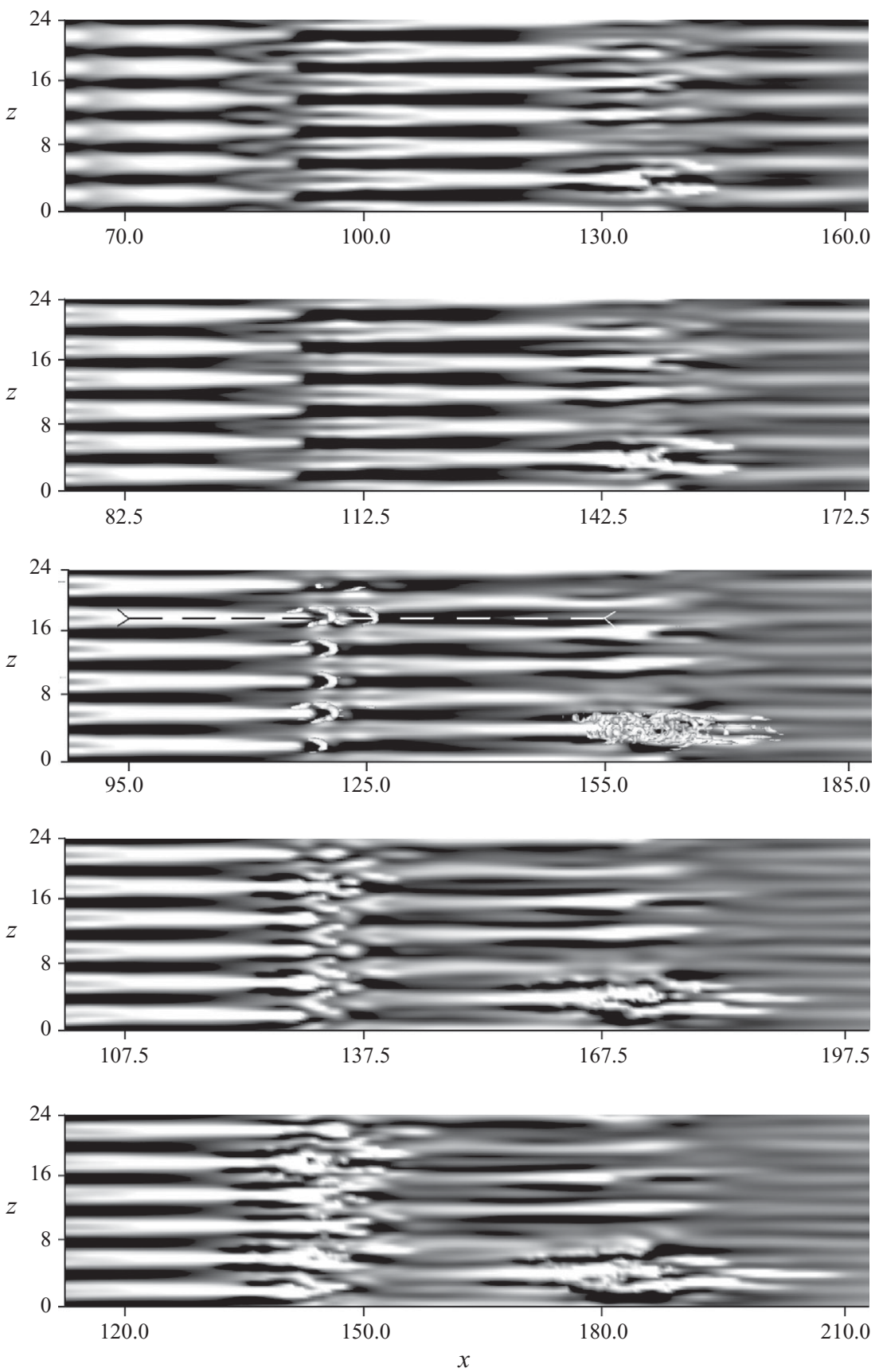

FIGURE 24. The $u$-perturbation velocity in the plane $y / \delta_{99}=0.25$, where $\delta_{99}$ is the boundary-layer thickness at $x=125$. Contour levels are $-0.10 \leqslant u \leqslant 0.10$. The sequence spans the period $T-100<t<T-20$, where $T$ is the time instance in figure 23 . The viewing window is translated at $0.625 U_{\infty}$ in order to follow the development of the instability.

eigenmode deviates significantly from the classical boundary-layer instability. It is no longer two-dimensional and, more importantly, is not monochromatic in time. Instead, it has finite energy over a range of frequencies, $200<F<600$. The higher 

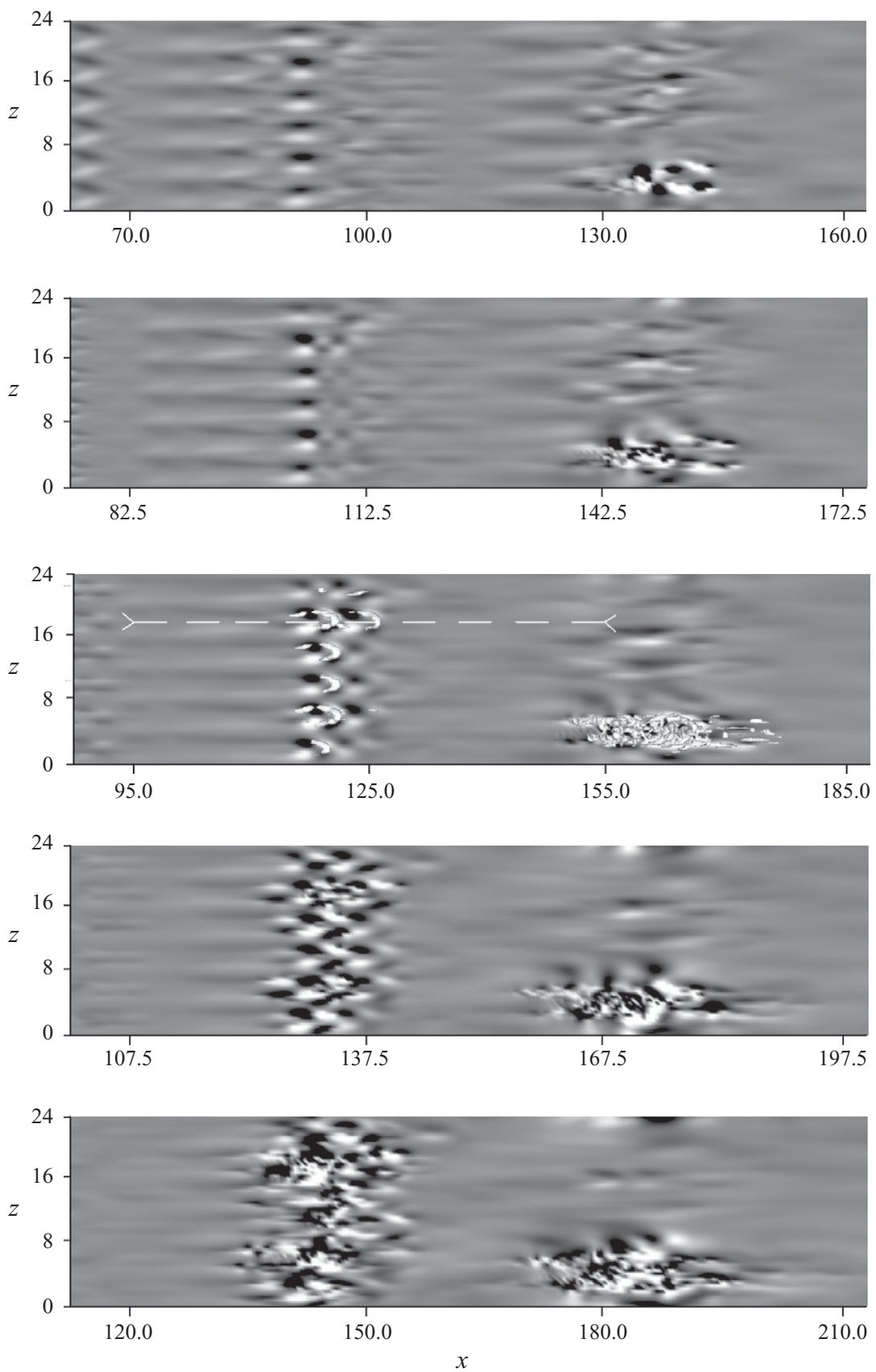

FIGURE 25. The $u$-perturbation velocity in the plane $y / \delta_{99}=0.25$, where $\delta_{99}$ is the boundary-layer thickness at $x=125$. Contour levels are $-0.05 \leqslant w \leqslant 0.05$. The sequence spans the period $T-100<t<T-20$, where $T$ is the time instance in figure 23 . The viewing window is translated at $0.625 U_{\infty}$ in order to follow the development of the instability.

frequencies are similar to the properties of the wave packets reported by Nagarajan et al. (2007; see their table 6).

A side view of the boundary layer at time $t=T-60$ is given in figure 26. The plane bisects the region where breakdown is initiated, and shows that the instability is near 


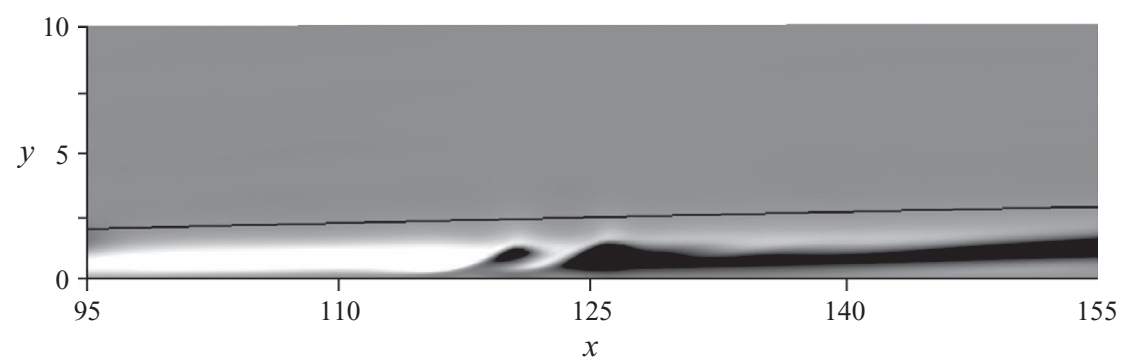

Figure 26. Contours of the $u$-perturbation velocity in the $(x, y)$ plane $(-0.1 \leqslant u \leqslant 0.1)$. Light and dark contours mark positive and negative velocity perturbations, respectively. The side view is extracted along the dashed line in figures 24 and 25.
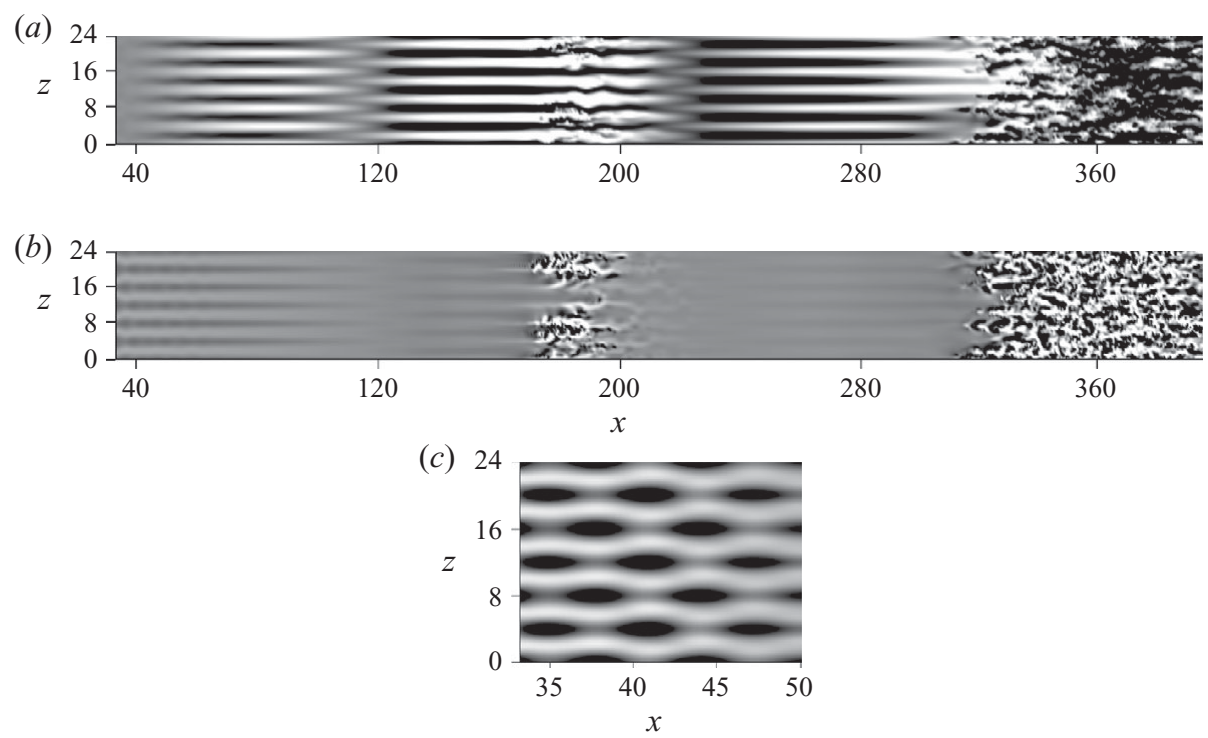

FIGURE 27. Contours of the perturbation velocities in the case of the outer mode: $(a)$ $-0.10 \leqslant u \leqslant 0.10 ;(b)-0.01 \leqslant v \leqslant 0.01 ;(c)$ a zoomed-in view of the region near the inlet of the computational domain, $-0.005 \leqslant v \leqslant 0.005$. The plane is $y=1.0$ based on the boundary-layer thickness at the inlet plane. Further downstream, $y / \delta_{99}=0.16$, where $\delta_{99}$ is the boundary-layer thickness at $x=360$.

the wall, at the intersection of the low- and high-speed streaks. The importance of this region was suggested by Brandt et al. (2004) based on their DNS. It was also noted by Zaki et al. (2010) in simulations of transition in compressor passages, where the overall pressure gradient is adverse and the interaction of streak and instability waves leads to breakdown to turbulence. However, a formal explanation of the instability mechanism was not provided.

\subsection{The outer mode}

Direct numerical simulations of streaks perturbed by the outer instability were also carried out. An overview of the downstream evolution of the flow, as computed from the DNS, is shown in figure 27. Transition to turbulence took place in the DNS at streak amplitudes of the order of $10 \%-15 \%$. This amplitude is significantly lower than the threshold for instability predicted by the steady analysis of Andersson et al. 

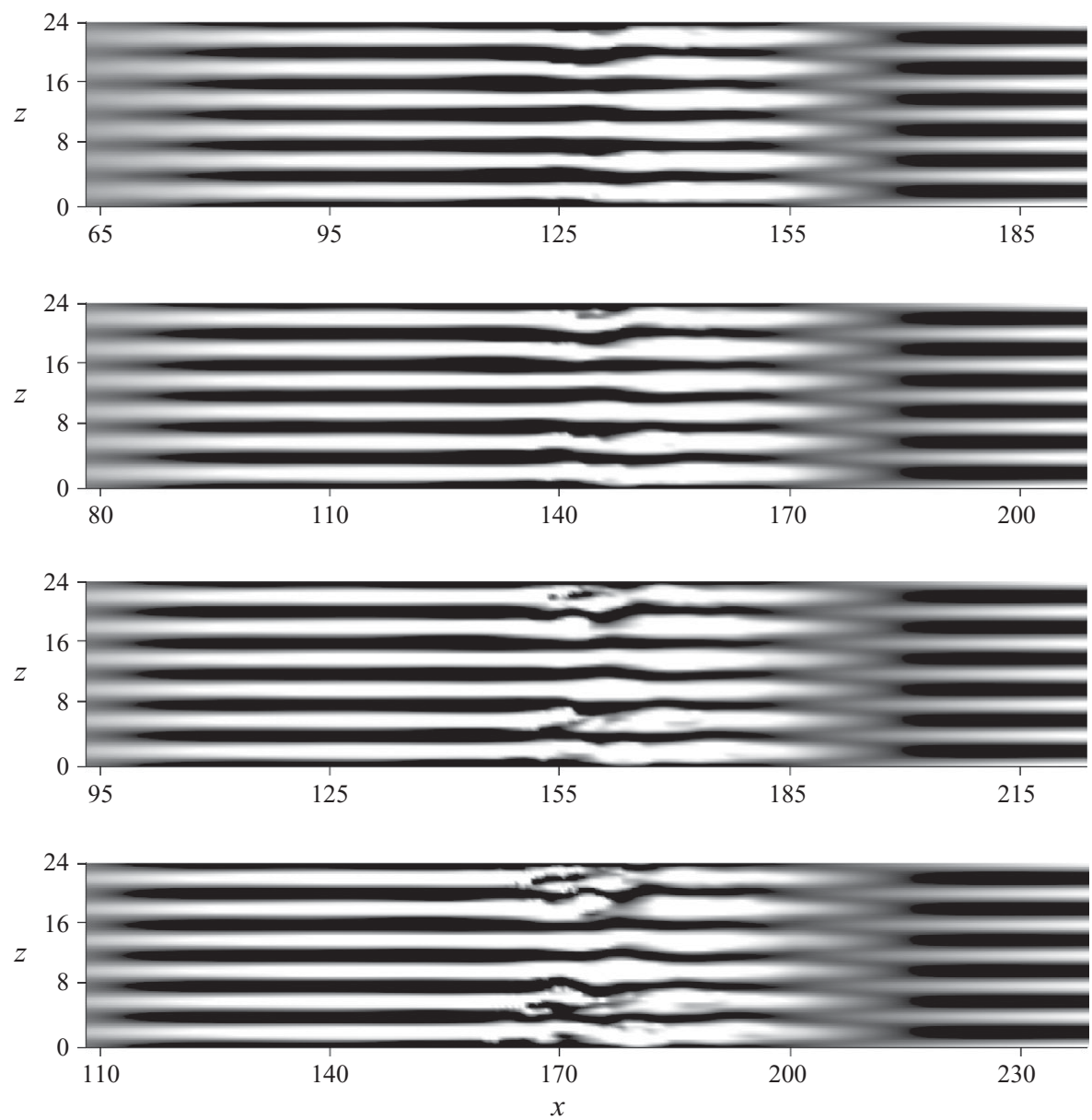

FIGURE 28. The $u$-perturbation velocity in the plane $y / \delta_{99}=0.38$, where $\delta_{99}$ is the boundary-layer thickness at $x=140$. Contour levels are $-0.10 \leqslant u \leqslant 0.10$. The sequence spans the period $T-80<t<T-20$, where $T$ is the time instance in figure 27 . The viewing window is translated at $0.75 U_{\infty}$ in order to follow the development of the instability.

(2001). The $u$-contours in the top pane of figure 27 clearly illustrate the streaks upstream of transition to turbulence. However, it is difficult to infer the presence of the outer mode. Therefore, the figure also shows a zoomed-in view of the region near the inlet of the domain, where $v$-contours are used to illustrate the spanwise subharmonic nature of the outer instability mode.

A time sequence of the amplification of the outer mode and breakdown of the streaky boundary layer is shown in figure 28 . The sequence spans $T-80<t<T-20$, where $T$ is the time instance in figure 27. The region shown translates downstream at $0.75 U_{\infty}$ in order to maintain the instability in the middle of the frame. The plane view of the perturbation field does not clearly demonstrate the outer nature of the instability. Also, using vortex identification criteria, such as $\lambda_{2}$, did not yield any distinctive vortical structures during the breakdown of the streaks.

Figure 29 shows a side view of the disturbance field at $t=T-60$. The plane corresponds to $z=8$ in figure 28 . The contours of $u$-perturbation show that the low-speed streak becomes unstable near the edge of the boundary layer, due to 


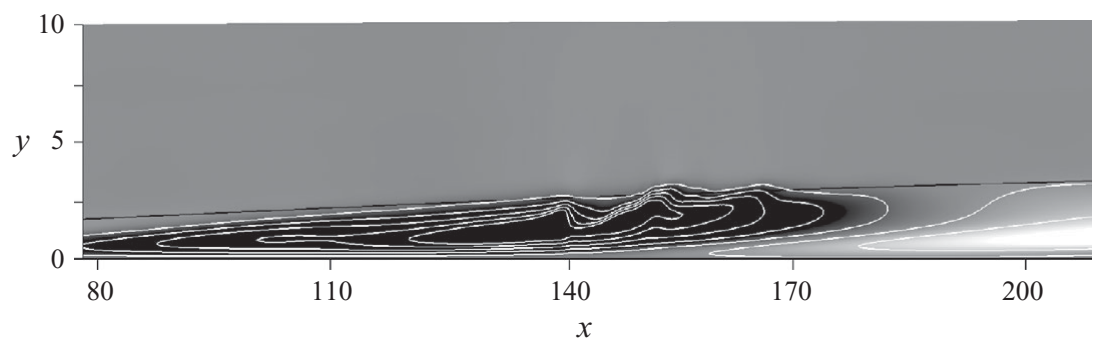

Figure 29. Contours of the $u$-perturbation velocity in the $(x, y)$ plane $(-0.1 \leqslant u \leqslant 0.1)$. Light and dark contours mark positive and negative velocity perturbations, respectively. The side view is extracted at $z=8$, and corresponds to time $t=T-60$, in figure 28 .

forcing by the outer mode. The instability very closely resembles the flow patterns reported by Zaki \& Durbin (2005), where their streak was perturbed by a highfrequency free-stream mode. It also bears a clear similarity to the flow patterns which preceded boundary-layer breakdown in previous simulations of bypass transition due to free-stream turbulence, in the absence of a leading edge (Jacobs \& Durbin 2001; Brandt et al. 2004). The mechanism was described by Jacobs \& Durbin (2001) as an irregularity which resembles a Kelvin-Helmholtz instability. Due to the localized appearance of the perturbation atop the streak, those authors could not qualify their observation as a streak instability. However, the results from the current DNS indicate that such a mechanism can be related to the outer mode, which is an instability mode of the streaky boundary layer.

\section{Conclusion}

The secondary instability of zero-pressure-gradient boundary layer, distorted by Klebanoff streaks, was investigated. The two most unstable modes were identified, and their growth rates were reported for a range of frequencies and amplitudes of the base streaks. The modes were classified as an 'inner' and an 'outer' instability, with reference to the position of their respective critical layers inside the boundary layer. The inner mode was related to the $\mathrm{T}-\mathrm{S}$ wave in the limit $A_{u} \rightarrow 0$, whereas the outer mode is a streak instability.

For moderate- and high-amplitude steady streaks, the nonlinear distortion of the mean velocity profile, $U_{0}$, stabilized the inner mode relative to the growth rate of $\mathrm{T}-\mathrm{S}$ waves. However, as the frequency of the streaks was increased, the wall-normal $u_{2}$ profile became locally inflectional, and the growth rate of the inner instability increased appreciably. This trend was most pronounced at higher levels of $A_{u}$ (figure 13). The growth rate of the inner mode was also reported at constant $A_{v}$, in order to account for the dependence of the base-streak amplitude on frequency, $F$. The results demonstrated that lower frequency, but not steady streaks, are the most destabilizing with respect to the inner mode (figure 15).

Given the location of the critical layer deep inside the shear, excitation of the inner instability is likely to depend on receptivity at the leading edge (Schrader $e t$ al. 2010). This view is supported by comparing our DNS of the inner instability to the simulations of Nagarajan et al. (2007). They investigated the influence of leadingedge bluntness and free-stream turbulence intensity on bypass transition. For high turbulence intensities and blunt leading edge, they observed the formation of nearwall wave packets which lead to breakdown to turbulence. Patterns similar to their 
$w$-perturbation contours, travelling at phase speed $c_{r} \approx 0.625 U_{\infty}$, were observed in the current DNS of boundary-layer breakdown due to the inner instability.

Based on the current results, the outer mode of instability emerged at a critical streak amplitude, $A_{u}^{c}=8.5 \%$. This threshold is commensurate with the levels reported in the literature on bypass transition due to free-stream turbulence. A comparison to the work of Andersson et al. (2001) was carried out by considering a steady base state, $u_{2}(y, z)$, and the influence of the streak ansatz on the stability threshold was discussed. In the case of a steady base flow, the outer mode is a sinuous and spanwise subharmonic instability. However, when the base streaks are unsteady, the sinuous and varicose modes coalesce. Nonlinear simulations of the outer instability were presented. Snapshots of the developing instability showed its origin near the free stream, prior to breakdown into a turbulent spot. Similar observations of secondary instability of streaks were reported based on previous simulations and physical experiments of bypass transition (Jacobs \& Durbin 2001; Hernon et al. 2007b).

In bypass transition, upstream of breakdown, the boundary layer contains a spectrum of low-frequency streaks, and is continually being perturbed by eddies from the free stream. Whether an inner or an outer instability emerges is dependent on the flow conditions. Relating the outer mode to previous observations of bypass transition, the turbulence in the free stream provides an effective forcing which can excite the high-frequency outer instability of the lifted streaks. This transition scenario was dominant in the DNS by Jacobs \& Durbin (2001), and the inner instability was not observed. Since the inner mode has a relatively low phase speed, and resides close to the wall, it is shielded from the high-frequency noise in the free stream by shear sheltering (Hunt 1977; Zaki \& Saha 2009). Instead, the inner instability can be excited more effectively due to receptivity at the leading edge, which was excluded from the simulation setup of Jacobs \& Durbin (2001). The effectiveness of this mechanism is dependent on the geometry and, in the presence of a blunt leading edge, the inner mode can provide an alternate bypass route to boundary-layer turbulence.

This work was supported by the UK Engineering and Physical Sciences Research Council (EPSRC).

\section{Appendix A. Fourier representation of the base streaks}

In the stability calculations, the base flow $u_{2}=U_{0}+u_{1}$ was represented by a truncated Fourier expansion,

$$
u_{1}(y, z, t)=\sum_{m=0}^{M} \sum_{n=0}^{N} \operatorname{Re}\left\{\hat{u}_{1}^{m, n}(y) \mathrm{e}^{\mathrm{i}\left(m \omega t+n k_{z} z\right)}\right\} .
$$

Only Fourier modes up to the second harmonic in time and in the span were retained, $M=N=2$. This was justified by comparing the energy captured by the truncated series to the total energy of the streaks in the DNS. The comparison was carried out at the target Reynolds number, and the $y$-location where the peak $u_{r m s}$ of the streak was recorded. The relative error in the energy was evaluated according to

$$
\mathscr{E}=1-\sum_{m=0}^{2} \sum_{n=0}^{2} E_{n, m} / E_{\text {total }},
$$

where $E_{n, m}$ is the energy content in the $(n, m)$ mode. Figure 30 shows the relative error for all streak frequencies and amplitudes. As the amplitude, and hence energy, 


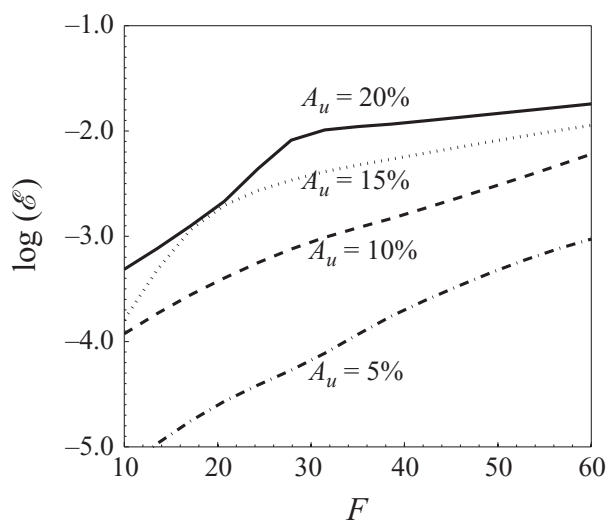

FIGURE 30. Relative error due to truncation of the Fourier series expansion of the base streak. The error is plotted for the range of streak frequencies and amplitudes considered in the stability analysis.

of the streak is increased, stronger nonlinear interactions emerge. As a result, higher harmonics are excited and the error in the truncation increases. However, the energy of the omitted modes remains approximately two orders of magnitude smaller than the total energy of the streak.

\section{Appendix B. Validation of the linear stability algorithm}

The secondary stability problem is derived by substituting the Floquet expansion (2.10) into the perturbation equations (2.6)-(2.8). This yields an eigenvalue problem, which is discretized using an expansion in Chebyshev polynomials (Orszag 1971). In operator form, the secondary instability modes are eigensolutions of

$$
A x=\sigma B x,
$$

where the eigenvalue is the frequency $\sigma$. The most unstable eigenvalues and the corresponding eigenvectors were computed using the implicitly restarted Arnoldi iteration method described by Lehoucq et al. (1998).

The base flows considered in this work can exhibit periodicity in time, in the spanwise direction, or in both coordinates simultaneously. Due to the linearity of the problem, periodicity in each coordinate direction can be validated independently.

\section{B.1. Time-periodic base flow: the oscillating plates problem}

The validation of the Floquet expansion in time was performed by comparing with the results of Blennerhassett \& Bassom (2006), who studied the linear stability of the time-periodic flow between two oscillating plates. In their work, the streamwise flow was driven by viscosity only, and therefore there is no pressure gradient. The analytic form for the time-dependent base flow provided by Blennerhassett \& Bassom (2006) was implemented in our Floquet solution algorithm. Stability calculations were performed at a plate separation of 32 units, where the wall-normal coordinate is scaled by $\sqrt{2 v / \omega}$, and $\omega$ is the frequency of wall oscillation. Three different Reynolds numbers were considered, $R e=\{570,700,750\}$. The spanwise wavenumber of the instability is a parameter; the value $a=0.3$ was selected for the validation tests.

Our results indicate that the flow is stable under these conditions. The decay rates of the least stable modes are compared to the results of Blennerhassett \& Bassom (2006) 


$\begin{array}{lccc}\text { Mode } & R e & \text { Blennerhassett \& Bassom (2006) } & \text { Current results } \\ \text { Even } & 570 & -0.06572 & -0.06572 \\ \text { Odd } & 570 & -0.11620 & -0.11620 \\ \text { Even } & 700 & -0.06676 & -0.06678 \\ \text { Odd } & 750 & -0.11951 & -0.12045\end{array}$

TABLE 1 . The growth rate of the least stable eigenvalue for oscillatory flow between two plates.

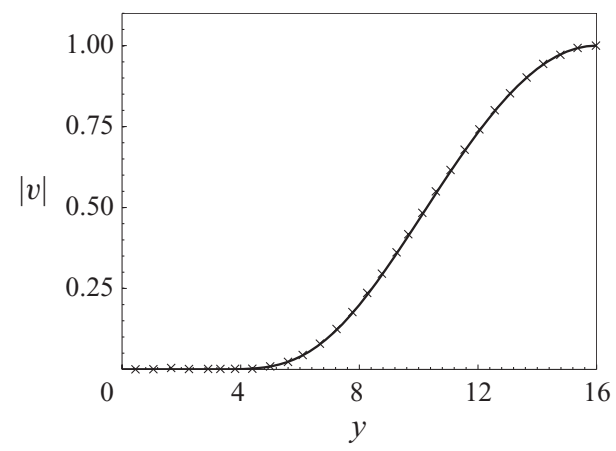

FIGURE 31. The normal velocity eigenfunction for the even mode at $R e=700$. - , current results; $\times$, Blennerhassett \& Bassom (2006).

in table 1 . The maximum relative error is below $1 \%$. In addition, figure 31 shows a comparison of our normal velocity eigenfunction and the results by Blennerhassett $\&$ Bassom (2006) for the even mode at $R e=700$. Excellent agreement is observed. Only four Floquet modes in the time expansion were sufficient. In the wall-normal expansion, 50 Chebyshev modes were used.

\section{B.2. Spanwise periodic base flow: boundary layer with steady streaks}

The validation of the spanwise Floquet expansion was previously carried out by Liu et al. (2008b). They computed the growth rates of Tollmien-Schlichting waves when the boundary layer is distorted by steady streaks, and compared their prediction to Cossu \& Brandt (2004). While the validation by Liu et al. (2008b) is sufficient, we herein present a further comparison against the work of Andersson et al. (2001) for steady streaks.

Andersson et al. (2001) studied the stability of zero-pressure-gradient boundary layer distorted by steady, spanwise periodic streaks. Their base flow streaks were obtained from the nonlinear evolution of the linearly optimal perturbation to the Blasius profile. Unlike the focus of Cossu \& Brandt (2004) on low-amplitude streaks and Tollmien-Schlichting waves, the work of Andersson et al. (2001) considered highamplitude Klebanoff modes which lead to an inflectional instability. Their base flow profile was also kindly provided by Dr L. Brandt for the purpose of this validation.

The amplification of the optimal streaks leads to appreciable mean flow distortion, as well as significant energy transfer to higher spanwise harmonics of the base streak. Therefore, 10 modes were required in the spanwise Floquet expansion for convergence of the stability results. Here, we focus on the growth rate of the most unstable modes, namely the subharmonic sinuous instability. Unlike the inviscid analysis by Andersson et al. (2001), our secondary stability equations (2.6)-(2.7) include viscous 


$\begin{array}{lcc}\text { Re } & \sigma_{r} & c_{r} \\ 100 & 0.01186 & 0.8693 \\ 200 & 0.01412 & 0.8633 \\ 300 & 0.01484 & 0.8619 \\ \text { Inviscid } & 0.01496 & 0.8450\end{array}$

TABLE 2. The effect of Reynolds number on the growth rate and wave speed of the subharmonic sinuous instability. The base flow used corresponds to $A=37.3$ in Andersson et al. (2001), and for comparison, the inviscid result comes from that paper.

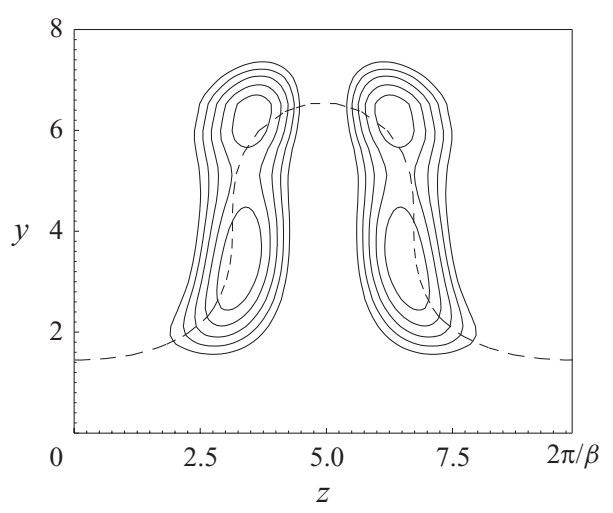

FiguRE 32. Contours of streamwise velocity perturbation in the subharmonic sinuous mode. The mode is normalized by its maximum absolute value, and levels of $|u|=[0.5,1.0]$ are plotted with a spacing of 0.1 . The base flow corresponds to the streak with $A=37.3 \%$ in Andersson et al. (2001). The dashed line denotes the distorted critical layer.

effects. Therefore, the Reynolds number in our analysis was gradually increased, and our predicted growth rates were compared to that of Andersson et al. (2001).

Table 2 compares our predicted growth rates and phase speeds at different Reynolds numbers to the inviscid results from Andersson et al. (2001). As the Reynolds number is increased, our results converge towards the inviscid value.

Streamwise velocity eigenfunctions were presented in Andersson et al. (2001, figure $12 b$ ), and demonstrated the distortion of the critical layer, particularly on the flanks of the low-speed streak. A similar plot is shown in figure 32 for the instability reported in table 2 , at $R e=300$. Although the contour levels were not specified in Andersson et al. (2001, figure 12b), and the streak amplitude for their figure is slightly lower than the value considered for this validation test, there is a strong qualitative agreement in the instability mode shape.

\section{REFERENCES}

Andersson, P., Brandt, L., Bottaro, A. \& Henningson, D. S. 2001 On the breakdown of boundary layer streaks. J. Fluid Mech. 428, 29-60.

Arnal, D. \& Juillen, J. C. 1978 Contribution expérimentale a l'étude de la réceptivité d'une couche limite laminaire, a la turbulence de l'écoulement general. Tech. Rep., ONERA Rap. Tech. 1/5018 AYD.

Asai, M., Minagawa, M. \& Nishioka, M. 2002 The instability and breakdown of a near-wall low-speed streak. J. Fluid Mech. 455, 289-314. 
Blennerhassett, P. \& BAssom, A. 2006 The linear stability of high-frequency oscillatory flow in a channel. J. Fluid Mech. 556, 1-25.

Boiko, A. V., Westin, K. J. A., Klingmann, B. G. B., Kozlov, V. V. \& Alfredsson, P. H. 1994 Experiments in a boundary layer subjected to free stream turbulence. Part 2. The role of TS-waves in the transition process. J. Fluid Mech. 281, 219-245.

BRAndT, L. \& Henningson, D. S. 2002 Transition of streamwise streaks in zero-pressure-gradient boundary layers. J. Fluid Mech. 472, 229-261.

Brandt, L., Sclatter, P. \& Henningson, D. S. 2004 Transition in boundary layers subject to free-stream turbulence. J. Fluid Mech. 517, 167-198.

Butler, K. M. \& Farrell, B. F. 1992 Three-dimensional optimal perturbations in viscous shear flow. Phys. Fluids A 4, 1637-1650.

Cossu, C. \& Brandt, L. 2002 Stabilization of Tollmien-Schlichting waves by finite amplitude optimal streaks in the Blasius boundary layer. Phys. Fluids 14 (8), 57-60.

Cossu, C. \& BRANDT, L. 2004 On Tollmien-Schlichting-like waves in streaky boundary layers. Eur. J. Mech. B/Fluids 23 (6), 815-833.

FASEL, H. F. 2002 Numerical investigation of the interaction of the Klebanoff-mode with a TollmienSchlichting wave. J. Fluid Mech. 450, 1-33.

Goldstein, M. E. \& Wundrow, D. W. 1995 Interaction of oblique instability waves with weak streamwise vortices. J. Fluid Mech. 284, 377-407.

Goldstein, M. E. \& Leib, S. J. 1993 Three-dimensional boundary-layer instability and separation induced by small-amplitude streamwise vorticity in the upstream flow. J. Fluid Mech. 246, $21-41$.

Goldstein, M. E. \& Sescu, A. 2008 Boundary-layer transition at high free-stream disturbance levels: beyond Klebanoff modes. J. Fluid Mech. 613, 95-124.

Grosch, C. \& Salwen, H. 1968 The stability of steady and time-dependent plane Poiseuille flow. J. Fluid Mech. 34, 177-205.

Herbert, T. 1988 Secondary instability of boundary layers. Annu. Rev. Fluid Mech. 20, 487-526.

Hernon, D., Walsh, E. J. \& McEligot, D. M. 2007a Instantaneous fluctuation velocity and skewness distributions upstream of transition onset. Intl J. Heat and Fluid Flow 28 (6), $1272-1279$.

Hernon, D., Walsh, E. J. \& McEligot, D. M. $2007 b$ Experimental investigation into the routes to bypass transition and the shear-sheltering phenomenon. J. Fluid Mech. 591, 461-479.

Hultgren, L. S. \& Gustavsson, L. H. 1981 Algebraic growth of disturbances in a laminar boundary layer. Phys. Fluids 24, 1000-1004.

Hunt, J. C. R. 1977 A review of rapidly distorted turbulent flows and its applications. Fluid Dyn. Trans. 9, 121-152.

Jacobs, R. G. \& Durbin, P. A. 2001 Simulations of bypass transition. J. Fluid Mech. 428, 185-212.

Kendall, J. M. 1990 Boundary layer receptivity to free stream turbulence. AIAA Paper 90-1504.

Kendall, J. M. 1991 Studies on laminar boundary-layer receptivity to freestream turbulence near a leading edge. In Boundary Layer Stability and Transition to Turbulence (ed. D. C. Reda, H. L. Reed \& R. Kobayashi), vol. 114, pp. 23-30. Fluid Engineering Division, ASME.

KerczeK, V. C. \& Davis, S. H. 1974 Linear stability theory of oscillatory Stokes layers. J. Fluid Mech. 62, 753-773.

Klebanoff, P. S. 1971 Effect of freestream turbulence on the laminar boundary layer. Bull. Am. Phys. Soc. 16 (11), 1323.

KLEISER, L. \& ZANG, T. A. 1991 Numerical simulation of transition in wall-bounded shear flows. Annu. Rev. Fluid Mech. 23, 495-537.

LANDAHL, M. T. 1980 A note on an algebraic instability of inviscid parallel shear flows. J. Fluid Mech. 98, 243-251.

LehoucQ, R., Sorensen, D. \& YAng, C. 1998 In ARPACK Users' Guide. SIAM Philadelphia.

Leib, S., Wundrow, D. \& GoldsteIn, M. 1999 Effect of free-stream turbulence and other vortical disturbances on a laminar boundary layer. J. Fluid Mech. 380, 169-203.

LiU, Y., ZAKi, T. A. \& DuRbin, P. A. $2008 a$ Boundary-layer transition by interaction of discrete and continuous modes. J. Fluid Mech. 604, 199-233.

LiU, Y., ZAKI, T. A. \& DURbin, P. A. 2008b Floquet analysis of secondary instability of boundary layers distorted by Klebanoff streaks and Tollmien-Schlichting waves. Phys. Fluids 20, 124102. 
Luo, J. \& WU, X. 2010 On the linear instability of a finite Stokes layer: instantaneous versus Floquet modes. Phys. Fluids 22, 054106.

Mandal, A. C., Venkatakrishnan, L. \& Dey, J. 2010 A study of boundary layer transition induced by freestream turbulence. J. Fluid Mech. 660, 114-146.

Mans, J., Kadijk, E. C., Lange, H. C. \& Steenhoven, A. A. 2005 Breakdown in a boundary layer exposed to free-stream turbulence. Exp. Fluids 39 (6), 1071-1083.

Mans, J., de Lange, H. C. \& van Steenhoven, A. A. 2007 Sinuous breakdown in a flat plate boundary layer exposed to free-stream turbulence. Phys. Fluids 19 (8), 088101.

Matsubara, M. \& Alfredsson, P. 2001 Disturbance growth in boundary layers subjected to free-stream turbulence. J. Fluid Mech. 430, 149-168.

Morkovin, M. V. 1969 On the many faces of transition. In Viscous Drag Reduction, pp. 1-31. Plenum.

Morkovin, M. V., Reshotko, E. \& Herbert, T. 1994 Transition in open flow systems: a reassessment. Bull. Am. Phys. Soc. 39, 1882.

Nagarajan, S., Lele, S. K. \& Ferziger, J. H. 2007 Leading-edge effects in bypass transition. J. Fluid Mech. 572, 471-504.

Nolan, K. P., Walsh, E. J. \& McEligot, D. M. 2010 Quadrant analysis of a transitional boundary layer subject to free-stream turbulence. J. Fluid Mech. 658, 310-335.

Orszag, S. A. 1971 Accurate solution of the Orr-Sommerfeld stability equation. J. Fluid Mech. 50, 689-703.

Phillips, O. M. 1969 Shear-flow turbulence. Annu. Rev. Fluid Mech. 1, 245-264.

Reshotкo, E. 1976 Boundary-layer stability and transition. Annu. Rev. Fluid Mech. 8 (1), 311-349.

Rosenfeld, M., KwaK, D. \& VinokUR, M. 1991 A fractional step solution method for the unsteady incompressible Navier-Stokes equations in generalized coordinate systems. J. Comput. Phys. 94 (1), 102-137.

SARIC, W. S. 1994 Görtler vortices. Annu. Rev. Fluid Mech. 26, 379-409.

SARic, W. S., Reed, H. L. \& Kerschen, E. J. 2002 Boundary-layer receptivity to freestream disturbances. Annu. Rev. Fluid Mech. 34, 291-319.

Schrader, L.-U., Brandt, L., Mavriplis, C. \& Henningson, D. S. 2010 Receptivity to free-stream vorticity of flow past a flat plate with elliptic leading edge. J. Fluid Mech. 653, 245-271.

SweARINGEN, J. D. \& BlaCKwEldER, R. F. 1987 The growth and breakdown of streamwise vortices in the presence of a wall. J. Fluid Mech. 182, 255-290.

Westin, K. J. A., Boiko, A. V., Klingmann, B. G. B., Kozlov, V. V. \& Alfredsson, P. H. 1994 Experiments in a boundary layer subjected to free stream turbulence. Part 1 . Boundary layer structure and receptivity. J. Fluid Mech. 281, 193-218.

Wu, X. \& Choudhari, M. 2003 Linear and nonlinear instabilities of a Blasius boundary layer perturbed by streamwise vortices. Part 2 . Intermittent instability induced by long-wavelength Klebanoff modes. J. Fluid Mech. 483, 249-286.

ZAKi, T. A. \& Durbin, P. A. 2005 Mode interaction and the bypass route to transition. J. Fluid Mech. 531, 85-111.

ZAKI, T. A. \& Durbin, P. A. 2006 Continuous mode transition and the effects of pressure gradient. J. Fluid Mech. 563, 357-388.

ZAKI, T. A. \& SAHA, S. 2009 On shear sheltering and the structure of vortical modes in single and two-fluid boundary layers. J. Fluid Mech. 626, 111-148.

Zaki, T. A., Wissink, J. G., Rodi, W. \& Durbin, P. A. 2010 Direct numerical simulations of transition in a compressor cascade: the influence of free-stream turbulence. J. Fluid Mech. 665, 57-98. 